\title{
GPER/ $\beta$-Alanine Positive Interaction in The Dorsal Root Ganglion Uncovers Potential Mechanisms: Mediating Continuous Neuronal Sensitisation and Neuroinflammation Responses in Neuropathic Pain
}

\section{Zhenzhen Xu}

Wuhan Xiehe Hospital: Wuhan Union Hospital https://orcid.org/0000-0002-3145-5273

Wanli Xie

Wuhan Xiehe Hospital: Wuhan Union Hospital

\section{Yiqi Feng}

Wuhan Xiehe Hospital: Wuhan Union Hospital

\section{Yanting Wang}

Wuhan Xiehe Hospital: Wuhan Union Hospital

\section{Yuyao He}

Wuhan Xiehe Hospital: Wuhan Union Hospital

Lu Chen

Wuhan Xiehe Hospital: Wuhan Union Hospital

\section{Yue Xiong}

Wuhan Xiehe Hospital: Wuhan Union Hospital

\section{Xia Li}

Wuhan Xiehe Hospital: Wuhan Union Hospital

Jie Liu

Wuhan Xiehe Hospital: Wuhan Union Hospital

\section{Guoyang Liu}

Wuhan Xiehe Hospital: Wuhan Union Hospital

Qingping Wu (D wqp1968@126.com)

Huazhong University of Science and Technology

\section{Research}

Keywords: $\mathrm{G}$ protein-coupled estrogen receptor, $\beta$-alanine, Dorsal root ganglion, Neuroinflammation, Neuropathic pain

Posted Date: November 30th, 2021 
DOI: https://doi.org/10.21203/rs.3.rs-1090097/v1

License: (c) (1) This work is licensed under a Creative Commons Attribution 4.0 International License. Read Full License 


\section{Abstract}

Background: The pathogenesis of neuropathic pain and the reasons for the prolonged unhealing are still unknown. Increasing evidence suggests that estrogen sex differences play a role in pain sensitivity, but few studies focused on the role of estrogen receptor which maybe an important molecular component contributing to peripheral pain transduction. We aimed to investigate the impact of oestrogen receptors in nociceptive neuronal response in the dorsal root ganglion (DRG) and spinal dorsal horn using a spared nerve injury (SNI) rat model of chronic pain.

Methods: We used a class of estrogen receptors antagonists and agonists intrathecal (i.t.) administrated to male rats with SNI or normal rats to identify the main receptor. Moreover, we applied genes identified through genomic metabolic analysis to determine the key metabolism point and elucidate potential mechanisms mediating continuous neuronal sensitisation and neuroinflammation responses in neuropathic pain. The excitability of DRG neurons was detected using the patch clamp technique. Immunohistochemistry, Western blotting, qPCR and behavioral testing were used to assess the expressions, cellular distributions, and actions of main receptor and its related signaling molecules.

Results: Increasing the expression and function of G protein-coupled estrogen receptor (GPER), but not estrogen receptor-a (ERa) and estrogen receptor- $\beta(E R \beta)$, in the $D R G$, but not the dorsal spinal cord, contributed to SNI-induced neuronal sensitisation. Inhibiting GPER expression in the DRG alleviated SNIinduced pain behaviours and neuroinflammation by downregulating IL-1 $\beta$ and IL- 6 expression as well as restoring GABAa2 expression simultaneously. Additionally, the positive interaction between GPER and $\beta$ alanine, $\beta$-alanine accumulation enhances pain sensation and promotes chronic pain development.

Conclusion: GPER activation in the DRG causes a positive interaction of $\beta$-alanine with IL-1 $\beta$ and IL- 6 expression and represses GABAa2 involved in post-SNI neuropathic pain development. Blocking GPER and eliminating $\beta$-alanine in the DRG may prevent neuropathic pain development.

\section{Introduction}

There is increasing evidence of the critical roles of estrogen in the peripheral and central nervous systems[1-3]. Estrogen targets neurons to regulate neural development, plasticity, and neuroprotective actions in behavioural and cognitive functions[4]. Estrogen exerts its effects through its receptors, which are widely distributed in the nervous system $[5,6]$. However, there have been few studies on the role of estrogen receptors (ERs), which may be crucially involved in peripheral pain transduction. It remains unclear whether modulating ERs could be an effective therapeutic strategy for neuropathic pain. Anatomical studies have confirmed that pain perception results from nociceptive signals produced by nociceptive neuron activation in the peripheral sensory nerves[7]. Subsequently, the nociceptive signals are transduced via the dorsal root ganglion (DRG) neurons, which synapse to spinal dorsal horn (SDH) neurons and finally project to the thalamus and cerebral cortex[8]. Neuropathic pain is a clinically common chronic refractory pain syndrome with a global prevalence of approximately $7-10 \%[9,10]$. 
Neuropathic pain has a complex pathogenesis with an unclear molecular mechanism, which impedes its clinical treatment. Recent studies have demonstrated increased intracellular $\mathrm{Cl}^{-}$levels in the DRG neurons after sciatic nerve section or inflammation[11-13]. There has been extensive research on chloride channels in primary sensory neurons since chloride channel activation in sensory neurons may cause chloride efflux and depolarisation due to high intracellular chloride concentrations [14-16]. Anion channels, especially chloride channels, may be involved in neuropathic pain pathogenesis $[17,18]$. Numerous studies have investigated GABAa2-mediated analgesia in neuropathic pain, whose absence is a classic neuropathic pain sign, accompanied by neuroinflammation[19, 20]. Spared nerve injury (SNI) is the most recognised method for simulating neuropathic pain[21, 22]. Numerous studies on the pathogenesis, prevention, and treatment of neuropathic pain have suggested that the pain threshold is sex-specific $[23,24]$. Notably, ERs are present in the DRG and SDH. Few studies have investigated pain regulation by estrogen or ERs through ion channels. GPER is a potential 17 $\beta$-E2 target that is modulated by the ERs in breast cancer cells[25]. Further, oestradiol was found to modulate the efficacy of synaptic inhibition by decreasing the dwell time of $\mathrm{GABA}_{A}$ receptors at inhibitory synapses[26]; however, the receptors involved remain unclear. The possible function of ERs in pain modulation is further supported by the ubiquitous expression of ERs in nociceptive system neurons[27]. There have been no studies focusing on the estrogen effects on pain modulation or the role of ERs in mediating continuous neuronal sensitisation and neuroinflammation responses in neuropathic pain. Additionally, estrogen can exert its effects in the absence of ERs. This study aimed to identify the main ERs involved in neuropathic pain and determine its related genomics and metabolomics to explore the role of ER-related metabolites in neuropathic pain. Specifically, we aimed to examine the metabolic mechanism of ERs in pain and elucidate the potential relationship of ERs with neuronal sensitivity and neuroinflammation.

\section{Methods}

\subsection{Animals}

We purchased adult male Sprague-Dawley rats (8-10 weeks old, $180-200 \mathrm{~g}, \mathrm{n}=400$ ) from the Animal Centre of the Charles River (Charles River Laboratories, US). The use of animals was approved by The Institutional Animal Care and Use Committee at Tongji Medical College, Huazhong University of Science and Technology, China. The animals were housed in plastic boxes with controlled temperature $\left(24 \pm 2^{\circ} \mathrm{C}\right)$, humidity (40-50\%), and a 12:12 h light:dark cycle. We selected rats with relatively uniform and stable baseline responses to cold, mechanical, and hot stimuli. All protocols were approved by the Animal Ethics Committee of the Tongji Medical College, Huazhong University of Science and Technology (approval No. 2405) on December 30, 2020. Further, they were consistent with the Guidelines for the Care and Use of Laboratory Animals, published by the US National Institutes of Health.

\subsection{Surgical Procedure for Establishing a Neuropathic Pain Model through Spared Nerve Injury (SNI)}


We established a model of neuropathic pain using SNI as previously reported [28]. Experimental procedures were performed on animals under anaesthesia through intraperitoneal administration of 40 $\mathrm{mg} / \mathrm{kg}$ sodium pentobarbital (Sigma-Aldrich, St. Louis, MO, USA). Care was exercised to prevent infection and reduce the inflammation impact. The skin was cut to expose the sciatic nerve and its three terminal branches directly through the part formed by the biceps muscle: the lateral side, common fibular nerve, and tibial nerves. The tibial and common peroneal nerves were cut and ligated through SNI, while the sural nerve was preserved. Given that the common peroneal and tibial nerves are closely connected, we subsequently removed $3-5 \mathrm{~mm}$ of the distal nerve ends. Care was taken to avoid damaging the nearby sural nerve. All wounds were postoperatively irrigated with sterile saline and closed in layers.

Experimenters blinded to the treatments performed the behavioural tests, electrophysiologic recordings, and Western blot and immunohistochemical experiments. The primary outcome was the effect of pharmacologic and genetic interventions involving different ERs in the DRG and SDH on pain-related aversion. We did not use analgesics.

\subsection{Groups and Drug Intervention}

All male rats were randomly divided into groups containing 6-8 rats. Drugs were dissolved in $2 \%$ DMSO and injected through a catheter for $24 \mathrm{~h}$. Intrathecal catheters were implanted on SNI day 5 as previously described [29]. We purchased 1,3-Bis (4-hydroxyphenyl)-4-methyl-5-[4-(2-piperidinyl ethoxy) phenol]-1Hpyrazole dihydrochloride (MPP) and PHTPP from Tocris Bioscience (United Kingdom). We purchased (3aS*,4R*,9bR*)-4-(6-Bromo-1,3-benzodioxol-5-yl)-3a,4,5,9b-3H-cyclopenta [c] quinolone (G15); 4,4',4"'- (4propyl-[1 H]-pyrazole-1,3,5-triyl) trisphenol (PPT); 2,3- bis (4-hydroxyphenyl)-propionitrile (DPN); ( \pm )-1[(3aR*,4S*, 9bS*)-4-(6-bromo-1,3-benzodioxol-5-yl)-3a,4,5,9b-tetrahydro-3H-cyclopenta [c]quinolin-8-yl]ethanone; and dimethyl sulfoxide from Sigma (USA). MPP (1 mM), G15 (1 mM), PPT (1 mM), DPN (5 $\mathrm{mM})$, and $\mathrm{G} 1(5 \mathrm{mM})$ were dissolved in dimethyl sulfoxide. $\beta$-alanine $(10 \mathrm{mM})$ was dissolved in saline. Stock solutions of the drugs were diluted in normal saline or artificial cerebrospinal fluid. Briefly, a salinefilled sterile catheter was inserted through the intervertebral space at $\mathrm{L}_{5} / \mathrm{L}_{6}$; moreover, the tube tip was positioned at the lumbosacral spinal level. We excluded animals with postoperative hindlimb paralysis or paresis. Animals without movement disorders received $2 \%$ lidocaine through the catheter to verify the intraspinal location. Correct catheterisation was confirmed by immediate bilateral hindlimb paralysis (within $15 \mathrm{~s}$ ) lasting 20-30 min. We excluded animals that lacked these features. DRGs for patch clamp experiments were incubated with $\mathrm{G} 1, \mathrm{G} 15$, and $\beta$-alanine in vitro.

\subsection{Measurement of $\beta$-alanine}

BioVision's Alanine Assay Kit allows simple and sensitive detection of alanine. In the kit, alanine is converted to pyruvate, which is specifically detected based on proportional colour $(\lambda=570 \mathrm{~nm}$ : 0-10 $\mathrm{nmol}$ ) or fluorescence (Ex/Em 535/587 nm: $01 \mathrm{nmol}$ ) development. The serum alanine levels are 2476 $\mu \mathrm{g} / \mathrm{ml}(\sim 3-9 \mathrm{nmol} / 10 \mu \mathrm{l})[30]$. Briefly, tissues or cells $\left(1 \times 10^{6}\right)$ were homogenised in a $100 \mu \mathrm{l}$ Assay Buffer centrifuge to remove insoluble material at $13,000 \mathrm{~g}$ for $10 \mathrm{~min}$. Subsequently, we directly diluted $10-50 \mu \mathrm{l}$ of deproteinised serum samples in the Assay Buffer and added them to a 96-well plate at $50 \mu \mathrm{l} / \mathrm{well}$. Next, sufficient reagents were prepared according to the number of assays to be performed. We added $50 \mu \mathrm{l}$ 
reaction mix [containing assay buffer $(44 \mu \mathrm{l})$, alanine converting enzyme $(2 \mu \mathrm{l})$, alanine development mix $(2 \mu \mathrm{l})$, and alanine probe $(2 \mu \mathrm{l})]$ to each well containing alanine standard, test, and background control samples. They were mixed well and incubated for $60 \mathrm{~min}$ at $37^{\circ} \mathrm{C}$ with protection from light.

Subsequently, the optical density was measured at $570 \mathrm{~nm}$ in a microplate reader or fluorescence using

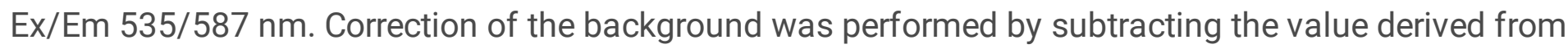
the alanine control sample from all the sample readings. This is because the background reading can be significant and must be subtracted from all sample readings. Finally, we plotted the alanine standard curve and calculated the alanine levels as follows: $\mathrm{C}=\mathrm{Sa} / \mathrm{Sv} \mathrm{nmol} / \mu \mathrm{l}$ or $\mathrm{mM}$.

\subsection{Behavioural Assays}

\subsubsection{Mechanical allodynia}

Mechanical allodynia was assessed by measuring the paw withdrawal threshold in response to a calibrated series of von Frey hairs (Stoelting) [31]. The rats were individually placed in cages with a wire mesh bottom. A series of calibrated von Frey hairs were applied to the plantar surface of the hind paw in an ascending order $(2,4,6,8,10,15$, and $26 \mathrm{~g})$ with sufficient force to bend the hair for 2 s or until paw withdrawal. Withdrawal responses were considered valid only if the hind paw was completely removed from the customised platform. Each hair was applied five times; moreover, the minimum value causing at least three responses was recorded as the paw withdrawal mechanical threshold (PWMT).

\subsubsection{Heat hyperalgesia (hot plate test)}

Thermal hyperalgesia was assessed as previously described [32]. The thermal withdrawal latency after radiant heat stimulation was measured using an analgesia meter (Ugo Basile, Stoelting, IL, USA). Animals were placed in the chamber and allowed to acclimatise for $30 \mathrm{~min}$ before testing. Subsequently, a radiant heat source was focused under the glass floor beneath the hind paws. We adjusted the thermal-stimulus intensity to obtain a baseline thermal withdrawal latency of approximately $20 \mathrm{~s}$. The digital timer automatically recorded the duration between stimulus initiation and the thermal withdrawal latency, with a 30-s cut-off being applied to prevent tissue damage. Each rat was tested at 5-min intervals, and the paw withdrawal thermal latency (PWTL) was calculated as the average of six trials.

\subsubsection{Cold allodynia (acetone drop method)}

Cold sensitivity was measured by applying an acetone drop to the plantar surface of the hind paw as previously described $[33,34]$. Rats were housed and habituated for $30 \mathrm{~min}$ in transparent plastic boxes using a wire-mesh floor. After the adaptation period, we gently applied acetone against the plantar skin of the left hind paws with an acetone bubble formed with a $0.1-\mathrm{ml}$ syringe alternately three times to hind paws at 5-min intervals. Moreover, we recorded the duration of licking/biting and remaining in the air. Each rat was tested at 5-min intervals, and the paw withdrawal cold duration (PWCD) was calculated as the average of six trials.

\subsection{Sample Preparation}


At predetermined time points after behavioural testing, the animals were deeply anaesthetised using sodium pentobarbital (40 mg/kg, intraperitoneal; Sigma) and sacrificed. Subsequently, ipsilateral $\mathrm{L}_{4-6}$ DRGs and SDH tissues were collected. Samples for reverse transcription polymerase chain reaction (RTPCR) and western blot experiments were snap-frozen in liquid nitrogen and stored at $-80^{\circ} \mathrm{C}$. We perfused samples used for immunofluorescence imaging through the ascending aorta with saline, followed by $4 \%$ paraformaldehyde in $0.1 \mathrm{M}$ phosphate buffer $\left(4^{\circ} \mathrm{C}, \mathrm{pH} 7.4\right)$, as previously reported [35].

\subsection{Immunofluorescence}

The L4-6 DRG on the surgical side was removed and fixed in $4 \%$ paraformaldehyde overnight, followed by dehydration in $20 \% / 30 \%$ sucrose in phosphate buffer at $4^{\circ} \mathrm{C}$. The tissue was cut into 5 - $\mu \mathrm{m}$ thick sections with a cryostat (Leica CM1950, Nussloch, Germany). The sections were blocked using $20 \%$ bovine serum albumin for $1 \mathrm{~h}$ in a $37^{\circ} \mathrm{C}$ incubator (303-0S; Beijing Ever Bright Medical Treatment Instrument Co., Ltd., Beijing, China), washed using phosphate-buffered saline (PBS), and incubated with primary antibody (rabbit anti-GPER polyclonal antibody; 1:100, 260033, Abcam, rabbit anti-ERa polyclonal antibody; 1:100, ab32063; Abcam, rabbit anti-ERß polyclonal antibody; 1:100, ab187291; Abcam) overnight at $4^{\circ} \mathrm{C}$. After washing with PBS, sections were incubated with secondary antibody (TRITCconjugated anti-rabbit secondary antibody; 1:100; Santa Cruz Biotechnology, Heidelberg, Germany) for 1 $\mathrm{h}$ at $37^{\circ} \mathrm{C}$. For double immunofluorescence staining, we incubated tissue sections with a mixture of antiGPER antibody and antibodies against neurofilament-200 (NF-200, a marker for myelinated A-fibres, 1:100, ab82259; Abcam, Cambridge, UK), calcitonin gene-related peptide (CGRP, a marker of peptidergic Ctype neurons, 1:100; ab81887; Abcam) for two nights at $4^{\circ} \mathrm{C}$ or IB4 (FITC-conjugated; a marker for nonpeptidergic C-type neurons, $5 \mu \mathrm{g} / \mathrm{ml}$; L2895; Sigma). Except for IB4-treated tissue sections, the other sections were treated using a mixture of FITC-and TRITC-conjugated secondary antibodies at a 1:100 dilution for $1 \mathrm{~h}$ at $37^{\circ} \mathrm{C}$. IB4 was 1:750 mixed using TRITC-conjugated secondary antibody. Sections were rinsed using 0.01 M PBS three times, mounted on gelatin-coated slides, and air dried. We visualised immunoreactivity using fluorescence microscopy; further, a negative control was used by omitting the primary antibody to confirm the immunoreaction specificity. Sections were observed at $200 \times$ magnification using a confocal laser scanning microscope (LSM710; Carl Zeiss AG, Oberkochen, Germany). Optical density measurements and data analysis of GPER-positive cells for both types of DRG neurons were performed using Image-Pro Plus 6.0 (Media Cybernetics, MD, USA). We recorded the percentage fluorescence results of the positive neurons of the three independent experiments.

\subsection{Western Blot Analysis}

We homogenised the frozen tissues and extracted the proteins using a nucleoprotein and cytoplasmic protein extraction kit (Keygen Biotech, Nanjing, China); further, $30 \mu \mathrm{g}$ of protein was mixed using sodium dodecyl sulphate sample buffer. Proteins were separated using standard sodium dodecyl sulphatepolyacrylamide gel electrophoresis (8-10\% gel) and transferred onto $0.45-\mu \mathrm{m}$ nitrocellulose membranes (Invitrogen, Carlsbad, CA, USA). We blocked membranes in $5 \%$ milk for $1 \mathrm{~h}$ and incubated them overnight at $4^{\circ} \mathrm{C}$ using the following primary antibodies: rabbit anti-GPER (polyclonal antibody; 1:1000, 260033, Abcam), rabbit anti-ERa (polyclonal antibody; 1:1000, ab32063; Abcam), rabbit anti-ERß (polyclonal 
antibody; 1:1000, ab187291; Abcam), rabbit anti-IL-6 (polyclonal antibody; 1:500, A2447; ABclonal), rabbit anti-IL-1 $\beta$ (polyclonal antibody; 1:500, A1112; ABclonal), rabbit anti- GABRa2 (polyclonal antibody; 1:100, A14185; ABclonal)and anti- $\beta$-actin (1:1000 dilution, ab8226, Abcam). The next day, we rinsed the membranes using tris-buffered saline Tween thrice for $10 \mathrm{~min}$ and incubated them using secondary antibodies (anti- rabbit immunoglobulin $\mathrm{G}$ against the primary antibodies). Staining was visualised using enhanced chemiluminescence (GE Healthcare, Chicago, IL, USA). We quantified band intensities using Image J software (Rawak Software Inc., Germany).

\subsection{Quantitative RT-PCR Analysis}

Total RNA was extracted from the ipsilateral $\mathrm{L}_{4-6}$ DRGs of rats using Trizol (Invitrogen) and reversetranscribed to cDNA using a qRT- PCR kit (Invitrogen) following the manufacturer's instructions [36]. For each cDNA target, 2- $\mu \mathrm{L}$ aliquots of each complete reverse transcriptase reaction were amplified in a $20-\mu \mathrm{L}$ reaction volume using SYBR Green Real Time PCR Master Mix (Toyobo Co. Ltd., Osaka, Japan) within 45 cycles of $95^{\circ} \mathrm{C}$ and $60^{\circ} \mathrm{C}$ for $12 \mathrm{~s}$ and $35 \mathrm{~s}$, respectively. Amplification was performed using the following primers: GPR30, 5'- CAAGCAGTCTTTCCGTCATGC-3' (forward) and 5'- CTGCTCCGTGCTGTCTGGTAT-3' (reverse); ERa, 5'- AGATCTTTGACATGTTGCTGGC-3' (forward) and 5'- CTCGGTGGATGTGGTCCTTC-3' (reverse); Erß, 5'- TGCAGCTCAACAGAGGACAGT-3' (forward) and 5'-TAGAACTTGGCATTCGGTGG-3' (reverse); GABRA1, 5'- GTGCGACCATAGAACCGAAAG-3' (forward) and 5'-AAGCGATTCTCAGTGCAGAGG-3' (reverse); GABRA2, 5'-GTTTATCGCTGTTTGTTACGCG-3' (forward); and 5'-TGTTCTGTATCATGACGGAGCC3' (reverse); GABRA3, 5'-CTTCACCAAGCGAAGTTGGG-3' (forward) and 5'-

GAGTTGAAGAAGCACTGGGAGC-3' (reverse); GABRA5, 5'- GCACAACATGACGACACCCA-3' (forward) and 5' -CAGACTTGGTGGAACCATTGG-3' (reverse); GABRB2, 5'- CCGTATGATTCGACTGCATCC-3' (forward) and 5' -GCTTTCGATCTCCAACGTGC-3' (reverse); GABRB3, 5'- TTCGTCTTTGTATTCCTGGCAC-3' (forward) and 5'GTGAACATCCATCGGTGCTAGT-3' (reverse); $\beta$-action, 5'-AGCAGA TGT GGATCAGCAAG-3' (forward) and 5' -AACAGTCCGCCTAGAAGCAT-3' (reverse). We used mRNA $\beta$-actin levels as an internal control; further, we obtained a standard curve to determine the relative levels of each cDNA target. We calculated the relative gene expression levels using the $2^{-(\Delta \Delta C t)}$ method. Triplicate analysis was performed for the expression level of each gene.

\subsection{Isolation of DRG Neurons}

We used enzyme digestion to dissociate $L_{4-6} D R G$ neurons from the ipsilateral side of the operation as previously described [35]. DRGs in the drug-intervention group were treated using $17 \beta$-oestradiol and Balanine. Briefly, we freed the excised ganglia from their connective tissue sheaths and cut them into pieces using a pair of sclerotic scissors in DMEM/F12 medium (GIBCO; Thermo Fisher Scientific, Waltham, MA, USA) under low temperature on ice. The fragments were transferred into $5 \mathrm{~mL}$ of DMEM/F12 medium containing trypsin $(0.4 \mathrm{mg} / \mathrm{mL}$, Sigma) and collagenase (type $1 \mathrm{~A}, 0.6 \mathrm{mg} / \mathrm{mL}$, Sigma), followed by incubation for $5 \mathrm{~min}$ at $37^{\circ} \mathrm{C}$. Subsequently, the ganglia were gently triturated using fine fire-polished Pasteur pipettes. The suspension was dissociated in DMEM/F12 medium and supplemented with $10 \%$ foetal bovine serum; moreover, DRG neurons were plated on glass cover slips 
coated using Poly-L-Lysine (Sigma). We maintained the cells in a humidified atmosphere $\left(5 \% \mathrm{CO}_{2}, 37^{\circ} \mathrm{C}\right)$ and used them for electrophysiological recordings 6-24 $\mathrm{h}$ after plating.

\subsection{Transcriptomics Studies Library Preparation for Transcriptome Sequencing}

DRG tissues of three rats in the sham, $\mathrm{SNI}$, and $\mathrm{SNI}+\mathrm{G} 15$ groups were randomly selected for transcriptome analysis. We extracted total RNA from approximately $150 \mathrm{mg}$ of DRG tissue using a MirVana total RNA extraction kit (Ambion, Carlsbad, CA, United States). RNA levels were measured using the Qubit ${ }^{\circledR}$ RNA Assay Kit and a Qubit $\circledast$ 2.0 Fluorometer (Life Technologies, Carlsbad, CA, United States). We assessed RNA integrity using an RNA Nano 6,000 Assay Kit of the Bioanalyzer 2,100 system (Agilent Technologies, CA, United States). We used $1.5 \mu \mathrm{g}$ of RNA per sample as the follow-up test material for RNA sample preparation. Whole transcriptome profiling was performed using NEBNext ${ }^{\circledR}$ Ultra ${ }^{\mathrm{TM}} \mathrm{RNA}$ Library Prep Kit for Illumina ${ }^{\circledR}$ (NEB, Ipswich, MA, United States) following the manufacturer's instructions.

\section{Clustering, Sequencing, and Quantification of Gene Expression}

We performed clustering of index-coded samples using a CBOT Cluster Generation system using HiSeq 4,000 PE Cluster Kit (Illumina). Next, we performed RNA sequencing (150 bp, pair-ends) using standard Illumina HiSeq 4000 platform protocols. All downstream analyses were based on high-quality clean data. Gene FPKM was calculated by adding the FPKM of each genome transcript. The differential expression of both conditions was analysed using DESeq2R software package (1.26.0). For genes with FPKM values $\geq 1$ in at least one sample, significant between-group differences in expression were determined according to the criteria as follows: $p<0.05$ and |log2FoldChangel $\geq 0.5$.

\section{Gene Ontology (GO) and KEGG Enrichment Analysis of Differentially Expressed Genes}

We used the Functional Annotation Bioinformatics Microarray Analysis (DAVID) database[37] to perform GO enrichment analysis of DEGs. GO terms $(p<0.05)$ were considered significantly enriched by DEGs. DEG enrichment in KEGG pathways was examined using KEGG Orthology Based Annotation System v3.0 software. P-values were calculated using one-way ANOVA based on the normalised dataset. KEGG terms $(p<0.05)$ were considered significantly enriched by DEGs.

\subsection{Metabolomics Analysis}

We randomly selected the DRG samples of 36 rats in the sham, SNI, and SNI+G15 groups for metabolomics analysis. Tissues were treated using methanol containing 2-chloro-L-phenylalanine (10 $\mu \mathrm{g} / \mathrm{ml})$ in a 4:1 ratio (methanol: plasma supernatant, $\mathrm{v} / \mathrm{v}, 250 \mu \mathrm{l})$. After centrifugation at $4^{\circ} \mathrm{C}(4,000 \mathrm{rpm}$, $10 \mathrm{~min}$ ), the supernatant was collected for analysis. We uniformly mixed a $50-\mu l$ aliquot of each centrifuged supernatant to yield a quality control (QC) sample. LC-MS analysis was performed using a 
Shimadzu UHPLC (ESI) system and an AB Sciex quadrupole time-of-flight mass spectrometer (TripleTOF 5600). All analyses were performed using the ACQUITY UPLC C18 column $(100 \mathrm{~mm} \times 2.1 \mathrm{~mm}, 1.7 \mu \mathrm{m}$, Waters). The mobile phase was a mixture of $0.1 \%$ formic acid in water $(A)$ and acetonitrile $(B)$. The proportion of mobile phase $B$ was as follows: $2-30 \%, 0-3 \mathrm{~min} ; 30-60 \%, 3-5 \mathrm{~min} ; 60-80 \%, 5-15 \mathrm{~min}$; 80-100\%, 15-16 min; 100\%, 16-19 min; 100-2\%, 19-20 min; and 2\%, 20-25 min. The injection volume, flow rate, and column temperature were $2 \mu \mathrm{l}, 0.3 \mathrm{ml} / \mathrm{min}$, and $40^{\circ} \mathrm{C}$, respectively. Time-of-flight mass spectrometry (TOF-MS) and TOFMS/ MS were synchronously performed. Mass spectra were acquired in the positive and negative ion modes. The parameters of the positive ion mode were as follows: ion spray voltage floating, $5,500 \mathrm{~V}$, and collision gas, $40 \mathrm{eV}$, while those in the negative ion mode were as follows: ion spray voltage floating, $-4,500 \mathrm{~V}$, and collision gas, $-40 \mathrm{eV}$. The turbo spray temperature was $550^{\circ} \mathrm{C}$, while the TOF-MS mass ranged from $\mathrm{m} / \mathrm{z} 50-1,250 \mathrm{Da}$. During analysis of the sample sequence, we run a QC sample after every five injections to validate the analytical methodology (Zhang et al., 2017). QC (n =6) and plasma samples were analysed using LC-MS. We evaluated data quality based on the relative SDs of the retention times and intensities of 10 typical peaks (including internal standard) of the QC samples.

\subsection{Electrophysiological Recordings}

All recordings were performed on small- and medium-diameter $(20-35 \mu \mathrm{m})$ neurons as previously described [38]. Coverslips with DRG neurons were mounted in a small flow-through chamber positioned on the stage of an inverted microscope (Nikon Eclipse Ti, Tokyo, Japan) to select DRG cells with smooth membrane surfaces and good translucency. We continuously perfused coverslips with gravity-driven bath solution. We performed standard whole-cell patch-clamp recordings of isolated DRG neurons at room temperature $\left(22^{\circ} \mathrm{C}\right)$ using an EPC-10 amplifier and the PULSE program (HKA Electronics, Lambrecht, Germany). Membrane capacitance was read from the amplifier using PULSE to measure the size of cells and current densities. Glass pipettes (3-5 M $\Omega$ ) were prepared using a Sutter P-87 puller (Sutter Instruments, Novato, CA, USA). Action potentials were elicited by a series of depolarising currents from 0 to $500 \mathrm{pA}(150 \mathrm{~ms})$ in 50-pA step increments under the current clamp mode to measure the current threshold (rheobase) in the vicinity of the explosive action potential current. The current was altered at 10pA steps, i.e., the minimal current that evoked an action potential, which is a parameter for excitability. The recorded signal was amplified using a MultiClamp 700B amplifier (Molecular Devices, LLC, Sunnyvale, CA, USA), filtered at $10 \mathrm{kHz}$, and converted by an Axon Digidata 1550A D/A converter (Molecular Devices) at a 10-20 kHz sampling frequency. Voltage errors were minimised using $80-90 \%$ series resistance compensation; further, we used linear leak subtraction for all recordings. For the current clamp experiments, the bath solution contained the following (in $\mathrm{mM}$ ): $140 \mathrm{NaCl}, 5 \mathrm{KCl}, 2 \mathrm{CaCl}_{2}, 2 \mathrm{MgCl}_{2}$, $10 \mathrm{D}$-glucose, and $10 \mathrm{HEPES}$. The $\mathrm{pH}$ was adjusted to $7.4 \mathrm{using} \mathrm{NaOH}$. The pipette solution contained the following (in mM): $30 \mathrm{KCl}, 100 \mathrm{~K}$-aspartate, $5 \mathrm{MgCl}_{2}, 2 \mathrm{Mg}$-ATP, $0.1 \mathrm{Na}$-GTP, and 40 HEPES, with adjustment of the $\mathrm{pH}$ to 7.2 using $\mathrm{KOH}$. All chemicals were obtained from Sigma. 


\subsection{Statistical Analysis}

All data are expressed as mean \pm SEM of three independent experiments. Prior to further statistical analysis, the normal distribution hypothesis of the test data and homogeneity of variance were examined. Statistical analyses were performed using SPSS 20.0 (SPSS Inc., Chicago, IL, USA) and Graphpad 9.0 (California, USA). We analysed PWMT, PWCD, and PWTL using repeated-measures analysis of variance. Multiple between-group comparisons at each time point were conducted using Bonferroni's post-hoc tests. Among-group comparisons of the western blot, PCR, and patch-clamp data were conducted using one-way analysis of variance followed by Tukey's post-hoc tests. Between-group comparisons were performed using Student's t-test. Statistical significance was set at $P<0.05$.

\section{Result}

3.1 Development of cold and mechanical allodynia in SNI rats had no effect on cold and thermal hypersensitivity. Time courses of the withdrawal latency to thermal stimuli were determined in the sham and SNI groups. Radiant heat stimulus (IR 50) was applied to the left paws of the rats. BTime courses of the withdrawal duration to cold stimuli were measured in the sham and SNI groups. Acetone $(100 \mu \mathrm{l})$ was used on the left hind paw of the rats. Time courses of withdrawal thresholds in the sham and SNI groups to von Frey hair stimuli on day 1 before and on days 3, 5, 7, 14, and 21 after the surgery. Mechanical stimuli were applied to the left hind paw of rats with von Frey hairs. (Fig. 1A), n=6, compared with the sham group at the same time point, ${ }^{* * *} \mathrm{p}<0.001$.

\subsection{CGRP loss in the SDH as well as co-expression of the three ERs in the missing area and mainly} expressed in pain-related small and medium-sized neurons on the SNI injury side. On the SNI injury side, there was significant CGRP loss in the SDH. (Fig. 1B, C). To determine the function of ERs in DRG neurons, we initially used immunofluorescence double staining to characterize the expression of ERs in DRG neurons. The percentages of IB4 (a marker of nonpeptidergic C-type neurons), CGRP (a marker of peptidergic C-type neurons)-,and NF-200 (a marker of A-type neurons). (Fig. 1D). Immunofluorescent double staining experiments showed that ERs colocalized with IB4, CGRP, and NF-200 (Fig. 1E). The percentages of IB4-, CGRP-, and NF-200-positive neurons relative to the percentage of ERs -positive cells respectively ( $n=6$ in each group; Fig. 1G). These results showed that ERs was mainly located in A- and Ctype neurons in the DRG. The neuronal diameter size ranges of IB4, CGRP, and NF-200 were $31.00 \pm 1.13$, $17.75 \pm 0.87$, and $42.75 \pm 1.917$, respectively ( $n=10$ in each group) (Fig. $1 F)$.

\subsection{ERa and GPER was significantly increased GPER and ERa expression in the DRG but not the SDH.} Immunofluorescence staining and PCR detection of rat ipsilateral L4-6 DRGs at spared nerve injury (SNI) showed that the protein and mRNA levels of GPER and ERa were higher than those in sham-operated rats on day 5 after the operation $(p<0.01)$ in each group (Fig. 2A and $3 C-E)$. Notably, the ERs fluorescence intensity protein and mRNA levels in SDH had no change. (Fig.s 2B, 3C-E).Thus, GPER and ERa is expressed at a higher level in small and medium-sized neurons associated with pain signaling in DRG.

(Fig.s 2B, 3C-E). 
3.4 G15 administration attenuated cold and mechanical allodynia in SNI rats. To evaluate the potential function of GPER in neuropathic pain, G15 was injected in day 5 of SNI. In all SNI + G15 group rats that received G protein-coupled estrogen receptor-1 antagonist (G15;1.8 $\mu \mathrm{g} / \mu \mathrm{l}, 1 \mu \mathrm{l})$, cold and mechanical allodynia induced by SNI was partially reversed, and the effect persisted last $24 \mathrm{~h}$ of behavioral testing. Estrogen receptor- $\alpha$ antagonist (MPP; $1 \mathrm{ng} / \mu \mathrm{l}, 1 \mu \mathrm{l}$ ) and estrogen receptor- $\beta$ antagonist (PHTPP; $1 \mathrm{ng} / \mu \mathrm{l}, 1$ $\mu \mathrm{l})$ did not affect PWMT and PWCD. (Fig. 4A, B).

\subsection{GPER can be involved in neuropathic pain by regulating a2-GABAA. Double immunofluorescence} staining showed that GPER and a2-GABAA are colocalized in the dorsal root ganglia, (Fig.s $3 \mathbf{G}$ ), indicated that GPER may be involved in the regulation of superficial sensations such as pain like a2-GABAA. a2GABAA in the DRG plays an important role in pathophysiology of NPP caused by sciatic nerve injury[39]. (Fig. 4F) GPER and ERa both upregulated post SNI operation, therefore, normal rats were administered with G1, PPT, and DPN, which are GPER-specific, ERa-specific, and ERß-specific agonists, respectively. Only GPER-specific agonists could induce hyperalgesia similar to the SNI model. (Fig.s 3A, B). SNI induced loss of GABAa2 restored after G15 in sheath. The mRNA levels were consistent with the western blotting results, which indicates that GPER can be involved in neuropathic pain by regulating a2-GABAA. (Fig.s 3F, 4C, D).

\subsection{Transcriptomics testing found that GPER involved in neuropathic pain regulation focus on metabolic pathways while regulating neuroinflammation. We examined the gene expression profiles with a focus on} mRNA in neurons of Sham, SNI, and SNI+G15 rats through RNA-Seq. A total of 18439 genes were expressed in the three groups (Fig. 5A). There are a total of 154 genes with differences between groups(Fig. 5B). Compared with the SNI+G15 group, 36 genes were up-regulated in the SNI group, and 24 genes were down-regulated, there were significant statistical differences(Fig. 5C, D). KEGG analysis of upregulated and down-regulated genes shows that the metabolic pathway has the most significant statistical difference(Fig. 5E, F). From this we can perform metabolomics testing on the three groups to find the key metabolites that cause GPER changes. At the same time, we unexpectedly discovered that GPER can regulate the expression of inflammatory factors IL- 6 and IL-1 $\beta$, and GPER blockers can significantly reduce neuroinflammation (Fig. 5G).

\subsection{Metabolomics tests showed that alanine could cause GPER upregulation. According to the results of} genomics, targeted metabolomics has detected more than 300 metabolites, and found that 14 substances have differences between groups, $\beta$-alanine, Aminocaproic acid, $1 \mathrm{H}$-Indole-3-acetamide, Nicotinic acid, Aminoadipic acid, N-Acetylglutamine, Acetic acid, alpha-Hydroxyisobutyric acid, Methylsuccinic acid, Lithocholic acid, L-Carnitine, 2,2-Dimethyladipic acid, 2,2-Dimethylsuccinic acid, Butyric acid (Fig. 6A, B). The Sham group compared with the SNI group, the SNI group compared with the $\mathrm{SNI}+\mathrm{G} 15$ group, the substances that have changed are $\beta$-alanine and Nicotinic acid (Fig. $6 \mathrm{C}$ ). We were especially interested in pathways involved in pain and inflammation responses, since these pathways may get involved in mediating neuroinflammation and pain mechanisms in SNI model rats. Therefore, we continued to perform PPI analysis of the metabolites that are related to process we identified from KEGG analysis, $\beta$-alanine may be a key factor causing the increase in GPER(Fig. 6D, E). 


\section{8 $\beta$-alanine and GPER can interact positively and co-cause the excitability of DRG neurons and neuroinflammation similar to SNI-induced. To further determine the contribution of $\beta$-alanine to the}

increased GPER in neuropathic pain, $\beta$-alanine $10 \mathrm{mM}$ or $1 \%$ DMSO as vehicle, $10 \mu \mathrm{L}$, was administered intrathecally to normal rats for $24 \mathrm{~h}$ mechanical and cold hyperalgesia, itching significantly altered in Rats (Fig. 7A). Post-SNI operation, the alanine content increased, and the alanine content continued to accumulate until 14 days later(Fig. 7D). After administration of GPER blocker, the content of alanine was significantly lower than that after $\mathrm{SNI}$, and the content of alanine was not reduced after administration of GABA, so it can be inferred that alanine is upstream of GABA (Fig. 7B). We speculated that there was an interaction between GPER and $\beta$-alanine. In normal rats, $\beta$-alanine content was significantly increased after administration of GPER agonist G1 (Fig. 7C). After the administration of $\beta$-alanine, the western-blot technique was used to detect a significant increase in the GPER protein content, which can confirm that there is a positive interaction between GPER and $\beta$-alanine, and a cascade effect is formed after SNI (Fig. 7E). Increased of GPER and accumulation of $\beta$-alanine simultaneously up-regulate downstream IL-6, IL$1 \beta$, and down-regulate GABA to cause hyperalgesia and neuroinflammation (Fig. 7F). Normal rats given GPER blockers G15 have no obvious effect on neuronal excitability and neuroinflammation, suggesting that GPER plays a role in pathological conditions (Fig. 7G). Temperature gradient experiments show that in DRG tissues incubated with $\beta$-alanine, GPER degrades more slowly, indicating that alanine can combine with GPER and play a role (Fig. 8). From a functional point of view, the patch clamp technique verified that the increase of GPER and $\beta$-alanine caused the excitability enhancement of DRG neurons similar to that induced by SNI (Fig. 7H, I), and revealed the pathogenesis of neuropathic chronic pain(Fig. 9).

\section{Discussion}

Currently, neuropathic pain cannot be effectively treated since its exact cause remains unclear[10, 40]. Sensitisation is crucial for neuronal hyperactivity and is characterised by overreacting to normal stimuli[41]. Ectopic discharge is the electrophysiological basis of allodynia; moreover, the change of ion channels is the main reason for abnormal discharges and a2/a3 GABAA-mediated analgesia in neuropathic pain[11, 42]. Increasing studies have shown that estrogen can affect pain by regulating ion channels; however, there are conflicting opinions regarding pain and analgesia[42]. Estrogen mainly acts through its three receptors GPER, ERa, and ER [5]. However, few studies have investigated the role of ER in peripheral pain transduction. We observed significant post-SNI GPER upregulation; moreover, blocking GPER can relieve hyperalgesia. These findings demonstrated the possible mechanism of neuropathic pain onset and persistence from a perspective of material metabolism.

Oestradiol modulates the efficacy of synaptic inhibition by decreasing the dwell time of GABAA receptors at inhibitory synapses[26]. Mouse models of inflammatory and neuropathic pain have revealed that the novel a2-GABAA receptor is antihyperalgesic [20]. Therefore, we chose other receptors as the reference molecular biological indicators of pain. In this study, after establishing the SNI model, there were behavioural changes in mechanical and cold hyperalgesia; moreover, there was no significant change in thermal pain. In the follow-up study, we chose PWMT and PWCD as the behavioural reference indicators. 
On the SNI injury side, there was significant CGRP loss in the SDH as well as co-expression of the three ERs in the missing area. Moreover, our findings demonstrated that GPER, ERa, and ER $\beta$ are mainly expressed in pain-related small and medium-sized neurons. DRG neurons of various sizes have different responses to various stimuli[43]. Small- and medium-sized neurons specifically respond to harmful thermal, chemical, and mechanical stimuli; therefore, they are considered multimodal nociceptors. Furthermore, the DRG and SDH are closely associated with pain transmission; moreover, we found that the three ERs are closely related to pain. Estrogen could influence P2X3 expression via the ERa and GPER ERa to affect neuropathic pain, which may be mediated through the ERK pathway [44]. Deleting the ER subunit a (ERa) in TRPV1 nociceptors abolishes IL-23- and IL-17-induced pain in females[45]. ER- $\beta$ and G protein-coupled ER-1, but not ER-a, in the rostral anterior cingulate cortex are involved in pain-related aversion by modulating N-methyl-D-aspartate receptor-mediated excitatory synaptic transmission[46]. Behavioural tests revealed that the PWMT and PWCD peaked on the fifth day and lasted > 14 days in SNI animals. Therefore, we choose the fifth day as the timepoint for selecting animals for the experiment and drug intervention. SNI animals have been reported to maintain strong mechanical allodynia throughout an 85-day observation period [47].

We used immunofluorescence to detect the expression of three ERs in DRG neurons and SDH areas with CGRP deletion. On the fifth day of SNI, there was significantly increased ERa and GPER expression in the DRG but not the SDH. Western blot and PCR results were consistent with those of immunofluorescence. G15, which is a specific blocker of GPER, can relieve hyperalgesia in SNI rats; however, MMP, which is a specific ERa blocker, cannot. Therefore, normal rats were administered with G1, PPT, and DPN, which are GPER-specific, ERa-specific, and ERß-specific agonists, respectively. Only GPER-specific agonists G1 could induce hyperalgesia similar to the SNI model. Our findings suggest that GPER could be involved in SNI-induced neuropathic pain development; moreover, GPER is an important molecular component contributing to peripheral pain transduction. A study using a rat model of visceral pain demonstrated that spinal ERa mediates oestradiol-induced pronociception [48]. The ER $\beta$ and ERa have specific advantages regarding analgesic effects[49]. Our findings suggest that GPER up-regulation in the DRG, but not the $\mathrm{SDH}$, may be an important component in SNI-induced neuropathic pain. The roles of GPER downstream signalling pathways remain unclear. This study provided evidence regarding chronic pain by using the a2GABAA receptor to evaluate pain behaviour and the role of GPER in pain. SNI allodynia is followed by downregulating the a2-GABAA receptor, which is improved by intrathecal G15 administration; moreover, mechanical pain and cold hyperalgesia are alleviated. Additionally, we tested the expression changes of other GABA subunits. The mRNA levels were consistent with the Western blotting results, which indicates that GPER can be involved in neuropathic pain by regulating a2-GABAA. Moreover, there was high a2GABAA and GPER expression on small and medium DRG neurons. Therefore, we will conduct follow-up research on GPER expression on the DRG.

We successfully established the rat SNI model and intrathecally administered GPER blockers. We examined the gene expression profiles with a focus on mRNA in neurons of SNI rats, sham rats, and SNI+G15 rats through RNA-Seq. We found several differentially expressed mRNAs. Further, we validated their expressions via the protein assay and examined the molecular functions, cellular components, and 
enriched biological processes of these DEGs by applying bioinformatics analysis. A sizeable proportion of these DEGs were exclusively involved in inflammatory processes; furthermore, IL- 6 and IL-1 $\beta$ are of significant characteristic significance. Injury to the peripheral sensory nerves causes a neuroinflammatory response in the somatosensory pathway, from the DRG to the spinal cord, which contributes to neuropathic pain[50]. Activating nociceptor sensory neurons through noxious stimuli triggers pain as well as increases capillary permeability and blood flow to yield neurogenic inflammation, which results from the antidromic activation of nociceptor peripheral terminals[51]. Additionally, ceRNA analysis further identified the direct or indirect regulatory relationship that may exist in the GPER metabolic pathway under SNI conditions.

Chronic pain, especially high-intensity chronic pain, is associated with an increased prevalence of cardiovascular risk factors and metabolic syndrome[52]. GPER upregulation is crucially involved in promoting neuroinflammation and DRG sensitisation during chronic pain. We performed metabolomic analysis in neurons of rats in all three groups. Compared with the sham and SNI+G15 groups, the SNI group showed changes in $\beta$-alanine and nicotinic acid. $\beta$-alanine showed the most significant changes through enrichment analysis among a series of metabolites. Further, administering G15, which is a GPER blocker, reverses the SNI-induced increase in alanine and alleviates behavioural problems. A local intradermal injection of $\beta$-alanine directly induces itching in humans, which confirms that $\beta$-alanine induces itching through a peripheral, cutaneous mechanism[53,54]. Additionally, certain mechanical stimuli mediated by recently discovered circuits contribute to the itch sensation in the spinal cord and brain. These circuits that mediate touch, pain, and itching could engage in cross modulation[55]. We administered $\beta$-alanine into the upper sheath of normal rats, and the behavioural test revealed that rats showed mechanical and cold hyperalgesia upon itching occurrence. After $\mathrm{SNI}$, there was a continuous increase in the $\beta$-alanine levels of the rat DRG until 14 days. Western blot experiments also revealed an increased GPER levels, increased inflammation, decreased a2-GABAA levels, and excitation of DRG neurons after SNI. The high excitability state of DRG neuron cells after the G15 SNI model can be relieved. Moreover, there was enhanced excitability of DRG neurons in normal rats after incubation with G1 and $\beta$ alanine. Supplementation with $\beta$-alanine has been shown to delay accumulation of lactate during exercise through buffering the formation of lactate from pyruvate[56]. In this study, we selected smalland medium-sized neurons. Therefore, our findings demonstrate that the post-SNI GPER upregulation in small DRG neurons increases the levels of the inflammatory cytokines IL-1 $\beta$ and IL-6, as well as decreases a2-GABAA levels. In addition, $\beta$-alanine accumulation can be reversed through $\mathrm{G} 15$ administration. Both the secretion of inflammatory factors and the firing of neurons require energy consumption[57]. It is also possible that the energy generated during the continuous accumulation of alanine may cause the DRG cells to continuously produce inflammation and nerve excitability. $\beta$-alanine can also positively regulate GPER formation similar to the waterfall effect. TGR7 functions as a specific membrane receptor for $\beta$-alanine, which corresponds to MrgD. Mas-related GPCR D is specifically expressed in small-diameter nociceptive DRG neurons and is implicated in pain modulation[58]. We confirmed that $\beta$-alanine could ligate the GPER, which could explain the chronicity of neuropathic pain. Notably, it remains unclear whether the effect of estrogen is the main contributor to sex differences in 
pain [59-61]. We can choose to locally deplete alanine and block GPER as an effective clinical treatment for chronic neuropathic pain

\section{Conclusions}

In conclusion, we speculate that GPER activation in the DRG causes a positive interaction of $\beta$-alanine with activation of IL-1 $\beta$ and IL- 6 production and represses GABAa2 involved in post-SNI neuropathic pain development. Therefore, GPER blocker and $\beta$-alanine elimination may be an underlying molecular target for controlling neuropathic pain without affecting normal sensation.

\section{Abbreviations}

GPER: G protein-coupled estrogen receptor; SDH: spinal dorsal horn; DMSO: dimethylsulfoxide; DRG: dorsal root ganglion; ER: estrogen receptor; PWMT: paw withdrawal mechanical threshold; PWCD: paw withdrawal cold duration; PWTL: paw withdrawal thermal latency; PBS: phosphate-buffered saline; SNI: spared nerve injury; RNA-Seq: RNA sequencing; DEmRNA: Differentially expressed mRNA; qPCR: Real-time quantitative PCR; KEGG: Kyoto Encyclopedia of Genes and Genomes; GO: Gene ontology; DEG:

Differentially expressed gene

\section{Declarations}

\section{Ethics approval and consent to participate}

This article does not contain any studies with human participants performed by any of the authors. All experimental procedures were carried out in accordance with the National Institutes of Health Guide for the Care and Use of Laboratory Animals (NIH publications no. 8023, revised 1978) and approved by the Animal Ethics Committee of Tongji Medical College, Huazhong University of Science and Technology (approval No. 2405) on December 30, 2020.

\section{Consent for publication}

Not applicable.

\section{Availability of data and materials}

The key data are contained in the figures, tables, and additional files. The datasets used and/or analyzed during this study can be further obtained from the corresponding author on reasonable request.

\section{Competing interests}

The authors declare that the research was conducted in the absence of any commercial or financial relationships that could be construed as a potential conflict of interest. 


\section{Funding}

This project was supported by the National Natural Science Foundation of China (Grant No. 81873952, Grant No. 81901948), the National Key Research and Development Program of China (Grant No. 2018YFC2001900). The funding sources had no role in study design, conception, analysis, or interpretation of data, writing, and deciding to submit this paper for publication.

\section{Authors' Contributions}

QPW, ZZX conceived and designed the experiments; ZZX and WLX conducted the experiments. YQF, XL, $\mathrm{JL}, Y X, Y Y H, L C, G Y L$ and helped with the experiments; ZZX and YTW analyzed the data. ZZX and QPW wrote the manuscript. All authors discussed and commented on the manuscript.

\section{Acknowledgments}

Not applicable.

\section{Authors' information}

${ }^{1}$ Department of Anaesthesiology, Union Hospital, Tongji Medical College, Huazhong University of Science and Technology, 1277 Jiefang Avenue, Wuhan 430022, China

\section{References}

1. Zhang QG, Wang R, Tang H, Dong Y, Chan A, Sareddy GR, Vadlamudi RK, Brann DW: Brain-derived estrogen exerts anti-inflammatory and neuroprotective actions in the rat hippocampus. $\mathrm{Mol} \mathrm{Cell}$ Endocrinol 2014, 389:84-91.

2. Xu ZZ, Chen QY, Deng SY, Zhang M, Tan CY, Yang W, Ma KT, Li L, Si JQ, Zhu LC: 17beta-Estradiol Attenuates Neuropathic Pain Caused by Spared Nerve Injury by Upregulating CIC-3 in the Dorsal Root Ganglion of Ovariectomized Rats. Front Neurosci 2019, 13:1205.

3. Huang X, Deng J, Xu T, Xin W, Zhang Y, Ruan X: Downregulation of metallothionein-2 contributes to oxaliplatin-induced neuropathic pain. J Neuroinflammation 2021, 18:91.

4. Yang JT, Wang ZJ, Cai HY, Yuan L, Hu MM, Wu MN, Qi JS: Sex Differences in Neuropathology and Cognitive Behavior in APP/PS1/tau Triple-Transgenic Mouse Model of Alzheimer's Disease. Neurosci Bull 2018, 34:736-746.

5. Liu JYH, Lin G, Fang M, Rudd JA: Localization of estrogen receptor ERa, ER $\beta$ and GPR30 on myenteric neurons of the gastrointestinal tract and their role in motility. General and Comparative Endocrinology 2019, 272:63-75.

6. Izquierdo P, Attwell D, Madry C: Ion Channels and Receptors as Determinants of Microglial Function. Trends Neurosci 2019, 42:278-292. 
7. Li HN, Yang QQ, Wang WT, Tian X, Feng F, Zhang ST, Xia YT, Wang JX, Zou YW, Wang JY, Zeng XY: Red nucleus IL-33 facilitates the early development of mononeuropathic pain in male rats by inducing TNF-alpha through activating ERK, p38 MAPK, and JAK2/STAT3. J Neuroinflammation 2021, 18:150.

8. Chen Q, Zhang W, Sadana N, Chen X: Estrogen receptors in pain modulation: cellular signaling. Biol Sex Differ 2021, 12:22.

9. de Moraes Vieira EB, Garcia JB, da Silva AA, Mualem Araujo RL, Jansen RC: Prevalence, characteristics, and factors associated with chronic pain with and without neuropathic characteristics in Sao Luis, Brazil. J Pain Symptom Manage 2012, 44:239-251.

10. Colloca L, Ludman T, Bouhassira D, Baron R, Dickenson AH, Yarnitsky D, Freeman R, Truini A, Attal N, Finnerup NB, et al: Neuropathic pain. Nature Reviews Disease Primers 2017, 3.

11. Lorenzo LE, Godin AG, Ferrini F, Bachand K, Plasencia-Fernandez I, Labrecque S, Girard AA, Boudreau D, Kianicka I, Gagnon M, et al: Enhancing neuronal chloride extrusion rescues alpha2/alpha3 GABAAmediated analgesia in neuropathic pain. Nat Commun 2020, 11:869.

12. Franco-Enzastiga U, Garcia G, Murbartian J, Gonzalez-Barrios R, Salinas-Abarca AB, SanchezHernandez B, Tavares-Ferreira D, Herrera LA, Barragan-Iglesias P, Delgado-Lezama R, et al: Sexdependent pronociceptive role of spinal alpha5 -GABAA receptor and its epigenetic regulation in neuropathic rodents. J Neurochem 2021, 156:897-916.

13. Dansereau MA, Midavaine E, Begin-Lavallee V, Belkouch M, Beaudet N, Longpre JM, MelikParsadaniantz S, Sarret P: Mechanistic insights into the role of the chemokine CCL2/CCR2 axis in dorsal root ganglia to peripheral inflammation and pain hypersensitivity. $J$ Neuroinflammation 2021, 18:79.

14. Bonin RP, De Koninck Y: Restoring ionotropic inhibition as an analgesic strategy. Neurosci Lett 2013, 557 Pt A:43-51.

15. Mao S, Garzon-Muvdi T, Di Fulvio M, Chen Y, Delpire E, Alvarez FJ, Alvarez-Leefmans FJ: Molecular and functional expression of cation-chloride cotransporters in dorsal root ganglion neurons during postnatal maturation. J Neurophysiol 2012, 108:834-852.

16. Chen Q, Kong L, Xu Z, Cao N, Tang X, Gao R, Zhang J, Deng S, Tan C, Zhang M, et al: The Role of TMEM16A/ERK/NK-1 Signaling in Dorsal Root Ganglia Neurons in the Development of Neuropathic Pain Induced by Spared Nerve Injury (SNI). Mol Neurobiol 2021.

17. Si J-Q, Li L, Chen Q-Y, Tan C-Y, Wang Y, Ma K-T: Mechanism of persistent hyperalgesia in neuropathic pain caused by chronic constriction injury. Neural Regeneration Research 2019, 14.

18. Wang L-J, Wang Y, Chen M-J, Tian Z-P, Lu B-H, Mao K-T, Zhang L, Zhao L, Shan L-Y, Li L, Si J-Q: Effects of niflumic acid on $Y$-aminobutyric acid-induced currents in isolated dorsal root ganglion neurons of neuropathic pain rats. Experimental and Therapeutic Medicine 2017, 14:1373-1380.

19. Witschi R, Punnakkal P, Paul J, Walczak JS, Cervero F, Fritschy JM, Kuner R, Keist R, Rudolph U, Zeilhofer HU: Presynaptic alpha2-GABAA receptors in primary afferent depolarization and spinal pain control. J Neurosci 2011, 31:8134-8142. 
20. Di Lio A, Benke D, Besson M, Desmeules J, Daali Y, Wang ZJ, Edwankar R, Cook JM, Zeilhofer HU: HZ166, a novel GABAA receptor subtype-selective benzodiazepine site ligand, is antihyperalgesic in mouse models of inflammatory and neuropathic pain. Neuropharmacology 2011, 60:626-632.

21. Szabo-Pardi TA, Barron LR, Lenert ME, Burton MD: Sensory Neuron TLR4 mediates the development of nerve-injury induced mechanical hypersensitivity in female mice. Brain Behav Immun 2021, 97:42-60.

22. Huang J, Zhang Z, Gambeta E, Chen L, Zamponi GW: An orbitofrontal cortex to midbrain projection modulates hypersensitivity after peripheral nerve injury. Cell Rep 2021, 35:109033.

23. Ramirez-Barrantes R, Marchant I, Olivero P: TRPV1 may increase the effectiveness of estrogen therapy on neuroprotection and neuroregeneration. Neural Regen Res 2016, 11:1204-1207.

24. Vacca V, Marinelli S, Pieroni L, Urbani A, Luvisetto S, Pavone F: 17beta-estradiol counteracts neuropathic pain: a behavioural, immunohistochemical, and proteomic investigation on sex-related differences in mice. Sci Rep 2016, 6:18980.

25. Yang H, Ma L, Wang Y, Zuo W, Li B, Yang Y, Chen Y, Chen L, Wang L, Zhu L: Activation of CIC-3 chloride channel by $17 \beta$-estradiol relies on the estrogen receptor $a$ expression in breast cancer. Journal of Cellular Physiology 2018, 233:1071-1081.

26. Mukherjee J, Cardarelli R, Cantaut-Belarif Y, Deeb T, Srivastava D, Tyagarajan S, Pangalos M, Triller A, Maguire J, Brandon N, Moss S: Estradiol modulates the efficacy of synaptic inhibition by decreasing the dwell time of GABA receptors at inhibitory synapses. Proceedings of the National Academy of Sciences of the United States of America 2017, 114:11763-11768.

27. Amandusson A, Blomqvist A: Estrogenic influences in pain processing. Front Neuroendocrino/2013, 34:329-349.

28. Xu N, Tang X-H, Pan W, Xie Z-M, Zhang G-F, Ji M-H, Yang J-J, Zhou M-T, Zhou Z-Q: Spared Nerve Injury Increases the Expression of Microglia M1 Markers in the Prefrontal Cortex of Rats and Provokes Depression-Like Behaviors. Frontiers in Neuroscience 2017, 11.

29. Pogatzki EM, Zahn PK, Brennan TJ: Lumbar catheterization of the subarachnoid space with a 32gauge polyurethane catheter in the rat. European Journal of Pain 2000, 4:111-113.

30. Homberg J, Wada T, Sameshima A, Yonezawa R, Morita M, Sawakawa K, Tsuneki H, Sasaoka T, Saito $\mathrm{S}$ : Impact of central and peripheral estrogen treatment on anxiety and depression phenotypes in a mouse model of postmenopausal obesity. Plos One 2018, 13.

31. Taccola G, Doyen PJ, Damblon J, Dingu N, Ballarin B, Steyaert A, Rieux Ad, Forget P, Hermans E, Bosier $B$, Deumens R: A new model of nerve injury in the rat reveals a role of Regulator of $G$ protein Signaling 4 in tactile hypersensitivity. Experimental Neurology 2016, 286:1-11.

32. Ouyang B, Chen D, Hou X, Wang T, Wang J, Zou W, Song Z, Huang C, Guo Q, Weng Y: Normalizing HDAC2 Levels in the Spinal Cord Alleviates Thermal and Mechanical Hyperalgesia After Peripheral Nerve Injury and Promotes GAD65 and KCC2 Expression. Front Neurosci 2019, 13:346.

33. Bergeson SE, Blanton H, Martinez JM, Curtis DC, Sherfey C, Seegmiller B, Marquardt PC, Groot JA, Allison CL, Bezboruah C, Guindon J: Binge Ethanol Consumption Increases Inflammatory Pain 
Responses and Mechanical and Cold Sensitivity: Tigecycline Treatment Efficacy Shows Sex Differences. Alcohol Clin Exp Res 2016, 40:2506-2515.

34. Deng L, Guindon J, Cornett BL, Makriyannis A, Mackie K, Hohmann AG: Chronic cannabinoid receptor 2 activation reverses paclitaxel neuropathy without tolerance or cannabinoid receptor 1-dependent withdrawal. Biol Psychiatry 2015, 77:475-487.

35. Zhang M, Gao CX, Wang YP, Ma KT, Li L, Yin JW, Dai ZG, Wang S, Si JQ: The association between the expression of PAR2 and TMEM16A and neuropathic pain. Molecular Medicine Reports 2017.

36. Sang Q, Sun D, Chen Z, Zhao W: NGF and PI3K/Akt signaling participate in the ventral motor neuronal protection of curcumin in sciatic nerve injury rat models. Biomed Pharmacother 2018, 103:1146-1153.

37. Huang da W, Sherman BT, Lempicki RA: Systematic and integrative analysis of large gene lists using DAVID bioinformatics resources. Nat Protoc 2009, 4:44-57.

38. Chen X, Pang R-P, Shen K-F, Zimmermann M, Xin W-J, Li Y-Y, Liu X-G: TNF-a enhances the currents of voltage gated sodium channels in uninjured dorsal root ganglion neurons following motor nerve injury. Experimental Neurology 2011, 227:279-286.

39. Obradovic A, Scarpa J, Osuru H, Weaver J, Park J, Pathirathna S, Peterkin A, Lim Y, Jagodic M, Todorovic S, Jevtovic-Todorovic V: Silencing the a2 subunit of Y-aminobutyric acid type A receptors in rat dorsal root ganglia reveals its major role in antinociception posttraumatic nerve injury. Anesthesiology 2015, 123:654-667.

40. Inoue $\mathrm{K}$, Tsuda M: Microglia in neuropathic pain: cellular and molecular mechanisms and therapeutic potential. Nature reviews Neuroscience 2018, 19:138-152.

41. Gilron I, Baron R, Jensen T: Neuropathic pain: principles of diagnosis and treatment. Mayo Clinic proceedings 2015, 90:532-545.

42. Finnerup N, Kuner R, Jensen T: Neuropathic Pain: From Mechanisms to Treatment. Physiological reviews 2021, 101:259-301.

43. Perl E: Ideas about pain, a historical view. Nature reviews Neuroscience 2007, 8:71-80.

44. Lu Y, Jiang Q, Yu L, Lu Z-y, Meng S-p, Su D, Burnstock G, Ma B: 17ß-Estradiol Rapidly Attenuates P2X3 Receptor-Mediated Peripheral Pain Signal Transduction via ERa and GPR30. Endocrinology 2013, 154:2421-2433.

45. Luo X, Chen O, Wang Z, Bang S, Ji J, Lee SH, Huh Y, Furutani K, He Q, Tao X, et al: IL-23/IL17AVTRPV1 axis produces mechanical pain via macrophage-sensory neuron crosstalk in female mice. Neuron 2021, 109:2691-2706 e2695.

46. Zang KK, Xiao X, Chen LQ, Yang Y, Cao QL, Tang YL, Lv SS, Cao H, Zhang L, Zhang YQ: Distinct Function of Estrogen Receptors in the Rodent Anterior Cingulate Cortex in Pain-related Aversion. Anesthesiology 2020, 133:165-184.

47. Norcini M, Sideris A, Martin Hernandez LA, Zhang J, Blanck TJ, Recio-Pinto E: An approach to identify microRNAs involved in neuropathic pain following a peripheral nerve injury. Front Neurosci 2014, 8:266. 
48. Ji Y, Tang B, Traub RJ: Spinal estrogen receptor alpha mediates estradiol-induced pronociception in a visceral pain model in the rat. Pain 2011, 152:1182-1191.

49. Coulombe MA, Spooner MF, Gaumond I, Carrier JC, Marchand S: Estrogen receptors beta and alpha have specific pro- and anti-nociceptive actions. Neuroscience 2011, 184:172-182.

50. Hu Z, Deng N, Liu K, Zhou N, Sun Y, Zeng W: CNTF-STAT3-IL-6 Axis Mediates Neuroinflammatory Cascade across Schwann Cell-Neuron-Microglia. Cell Rep 2020, 31:107657.

51. Michoud F, Seehus C, Schönle P, Brun N, Taub D, Zhang Z, Jain A, Furfaro I, Akouissi O, Moon R, et al: Epineural optogenetic activation of nociceptors initiates and amplifies inflammation. Nature biotechnology 2021, 39:179-185.

52. Goodson N, Smith B, Hocking L, McGilchrist M, Dominiczak A, Morris A, Porteous D, Goebel A: Cardiovascular risk factors associated with the metabolic syndrome are more prevalent in people reporting chronic pain: results from a cross-sectional general population study. Pain 2013, 154:1595-1602.

53. Liu Q, Sikand P, Ma C, Tang Z, Han L, Li Z, Sun S, LaMotte RH, Dong X: Mechanisms of itch evoked by beta-alanine. J Neurosci 2012, 32:14532-14537.

54. Seino $\mathrm{Y}$, Ohashi N, Kohno $\mathrm{T}$ : The endogenous agonist, beta-alanine, activates glycine receptors in rat spinal dorsal neurons. Biochem Biophys Res Commun 2018, 500:897-901.

55. Lay M, Dong X: Neural Mechanisms of Itch. Annu Rev Neurosci 2020, 43:187-205.

56. Vaughan R, Gannon N, Garcia-Smith R, Licon-Munoz Y, Barberena M, Bisoffi M, Trujillo K: $\beta$-alanine suppresses malignant breast epithelial cell aggressiveness through alterations in metabolism and cellular acidity in vitro. Molecular cancer 2014, 13:14.

57. Karagiannis A, Gallopin T, Lacroix A, Plaisier F, Piquet J, Geoffroy H, Hepp R, Naudé J, Le Gac B, Egger $R$, et al: Lactate is an energy substrate for rodent cortical neurons and enhances their firing activity. eLife 2021, 10.

58. Wang C, Gu L, Ruan Y, Geng X, Xu M, Yang N, Yu L, Jiang Y, Zhu C, Yang Y, et al: Facilitation of MrgprD by TRP-A1 promotes neuropathic pain. FASEB J 2019, 33:1360-1373.

59. Sarajari S, Oblinger MM: Estrogen effects on pain sensitivity and neuropeptide expression in rat sensory neurons. Exp Neurol 2010, 224:163-169.

60. Sorge RE, Totsch SK: Sex Differences in Pain. J Neurosci Res 2017, 95:1271-1281.

61. Chen S, Markman J, Shimada K, Crother T, Lane M, Abolhesn A, Shah P, Arditi M: Sex-Specific Effects of the NIrp3 Inflammasome on Atherogenesis in LDL Receptor-Deficient Mice. JACC Basic to translational science 2020, 5:582-598.

\section{Figures}


A
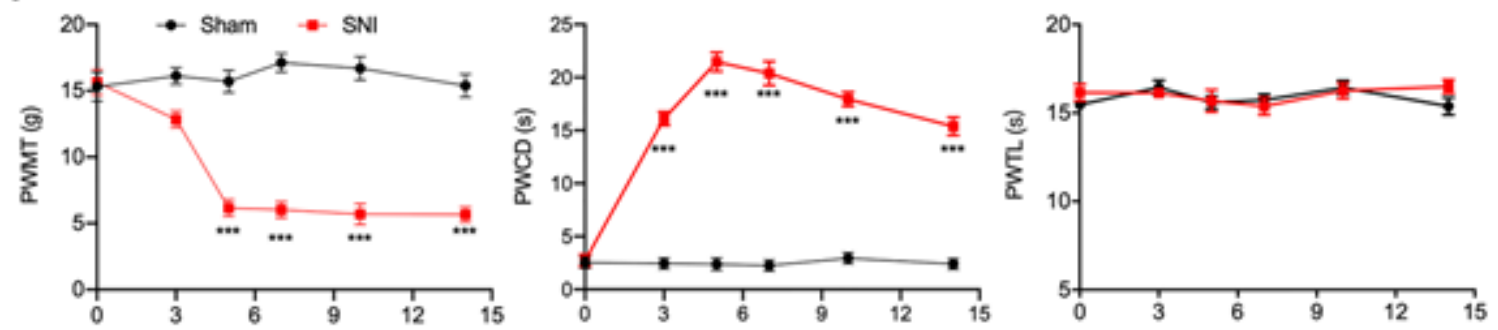

B

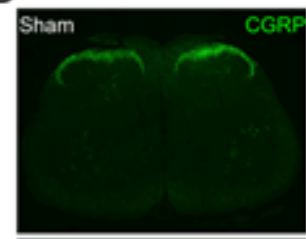

D

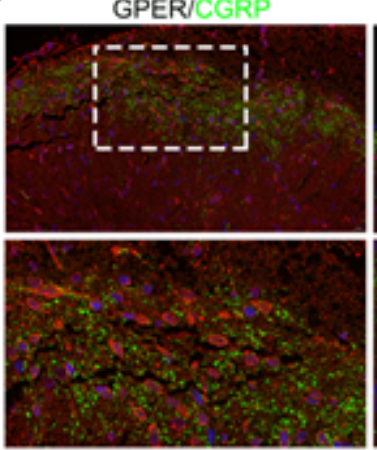

ER- $\alpha / C G R P$

ER- $\beta / C G R P$
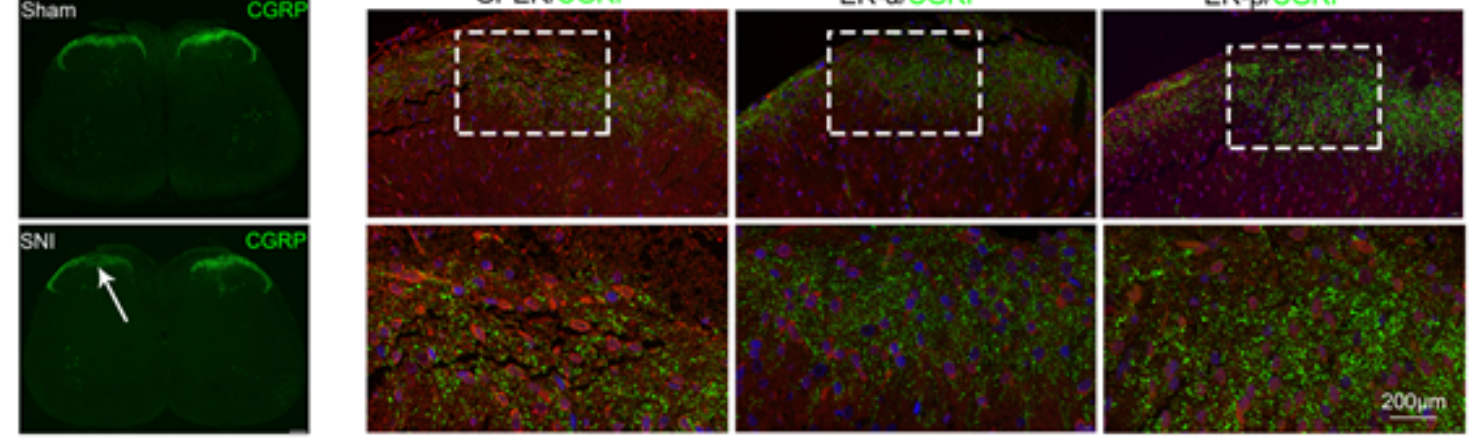

C
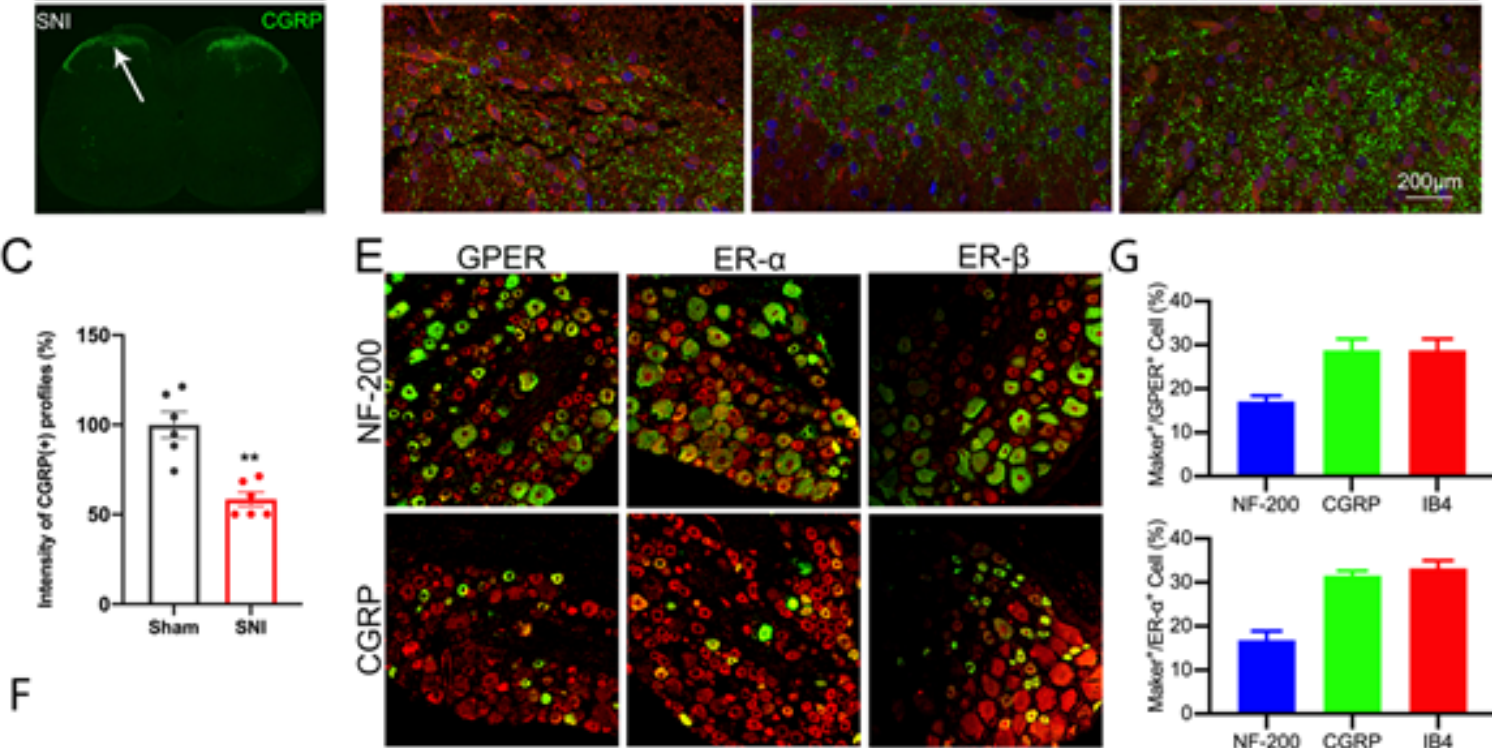

$\mathrm{F}$
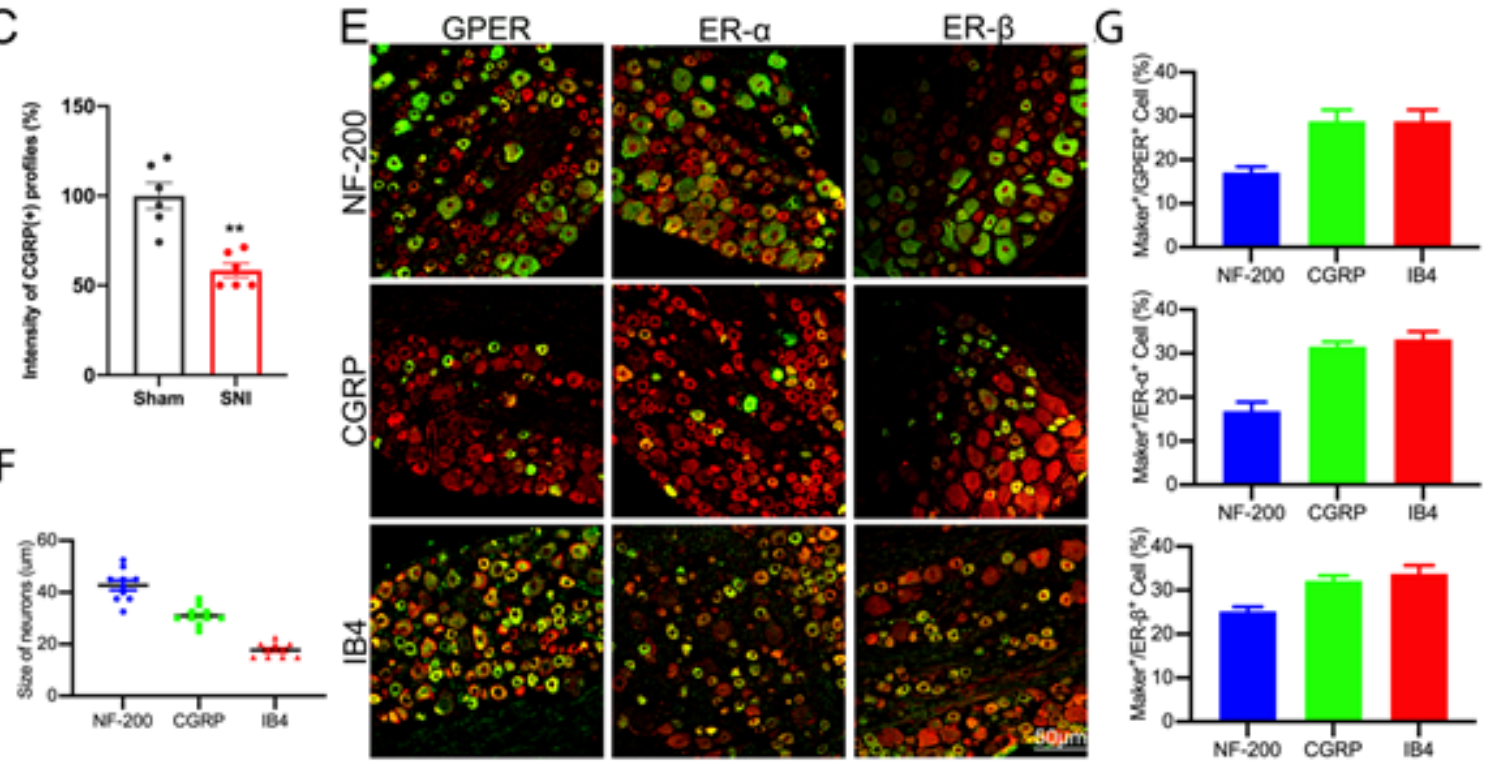

Figure 1

Distribution and expression of estrogen receptors (ERs) in the dorsal root ganglion (DRG) and dorsal spinal cord and spared nerve injury (SNI) model building. (A) Mechanical allodynia and cold hyperalgesia were developed in SNI rat paw compared to Sham rat since the second day after SNI until at least day 14, thermal hyperalgesia had no significant $(n=8)$. (B) Loss of $\operatorname{CGRP}(+)$ terminals in the ipsilateral SDH (arrow). (C) Quantification of the number of CGRP( + ) terminals in sham and SNI rats ( $n=7$ rats). (D) Double staining showing the colocalization of estrogen receptor-a, estrogen receptor- $\beta$, and $G$ proteincoupled estrogen receptor (green) with the CGRP (red) in the rat SDH. Scale bar, $200 \mu \mathrm{m}$. (E) Immunofluorescence double labeling revealed that the ERs was colocalized with IB4 (a marker of nonpeptidergic C-type neurons), CGRP (a marker of peptidergic C-type neurons), and NF-200 (a marker of A- 
type neurons). Arrows refer to co-labeled neurons, scale bar $=50 \mu \mathrm{m} .(\mathrm{F})$ Neuronal diameter size of IB4, CGRP, and NF-200. DRG: dorsal root ganglion. (G) The percentage of IB4, CGRP, and NF-200 positive (green) neurons relative to ERs (red) positive cells.

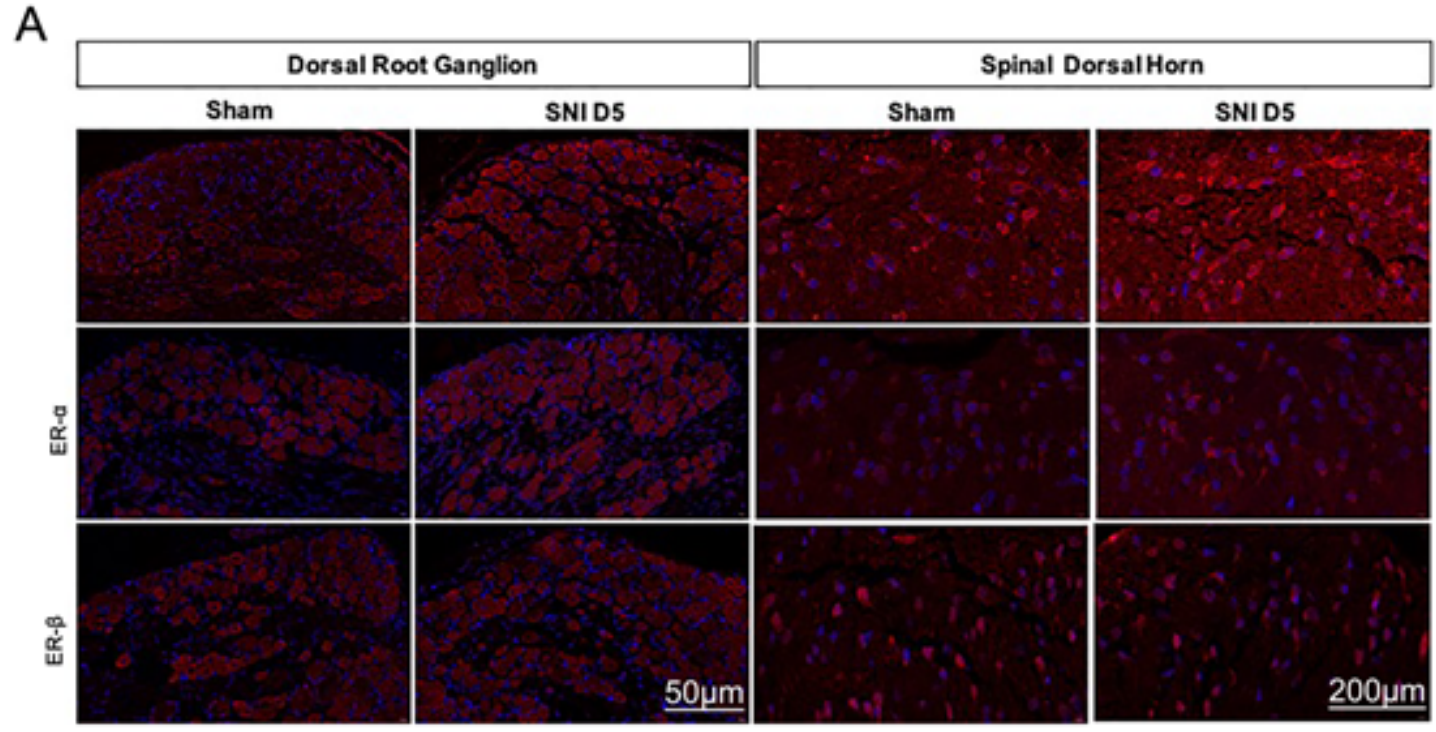

B DRG

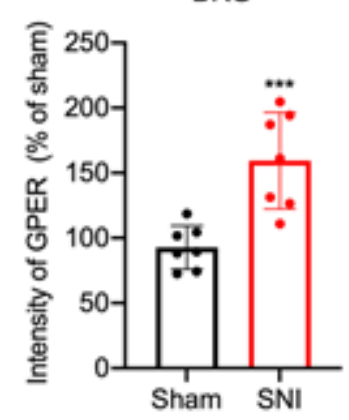

Spinal

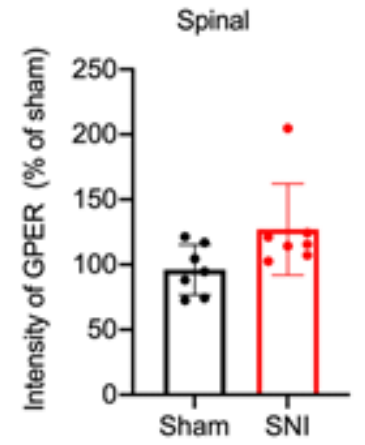

DRG

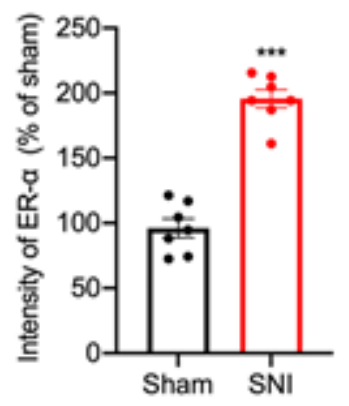

Spinal

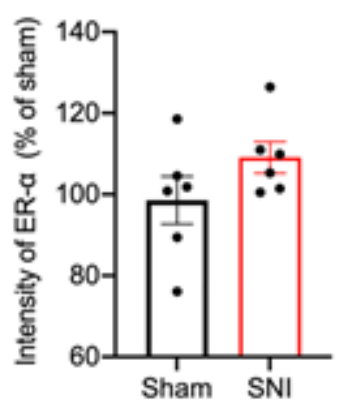

DRG

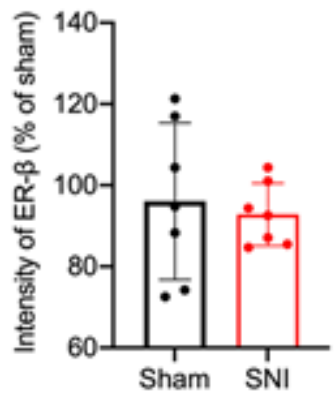

Spinal

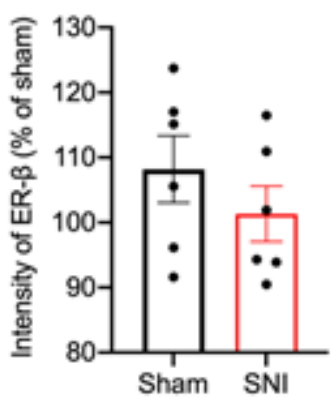

\section{Figure 2}

Expression of estrogen receptors (ERs) in the dorsal root ganglion (DRG) and dorsal spinal cord of spared nerve injury (SNI) rats. ( $A$ and $B)$ Representative images $(A)$ and quantifications $(B, n=6-7$ sections from three rats) showed the estrogen receptor- $a$, estrogen receptor- $\beta$, and $G$ protein-coupled estrogen receptor expression in DRG and dorsal spinal cord of SNI rat compared to Sham rat. ${ }^{\star \star \star} P<0.001$, ${ }^{\star \star} \mathrm{P}<0.01$ versus Sham. 
A

B
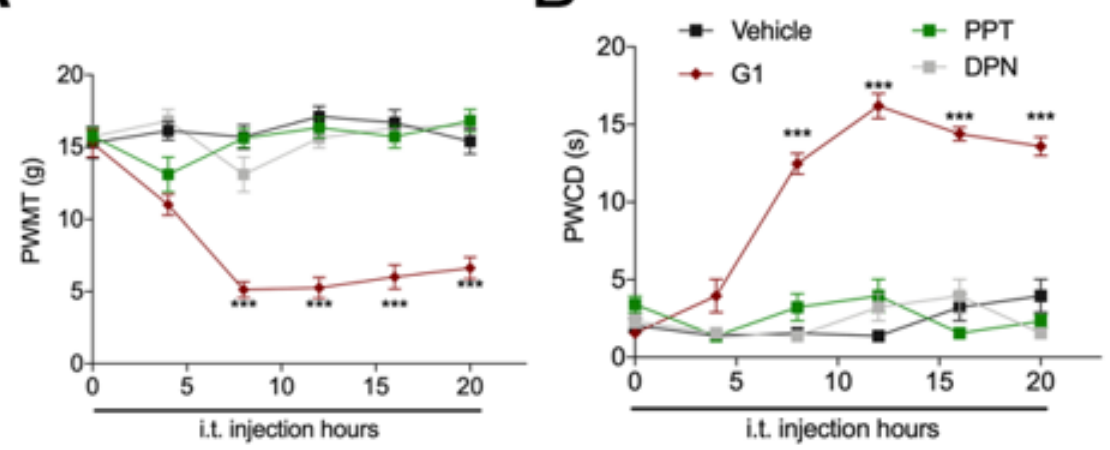

C

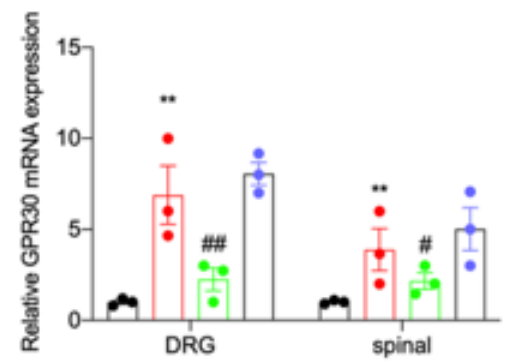

D
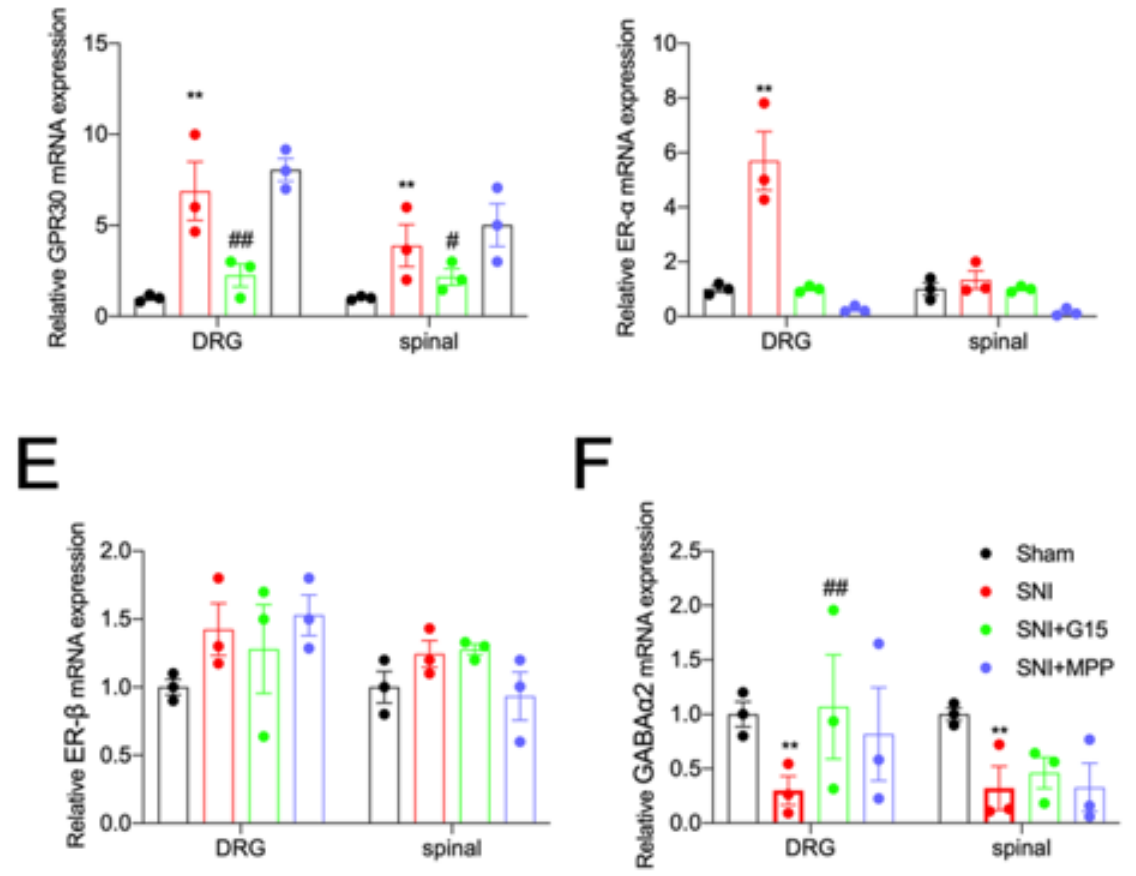

G
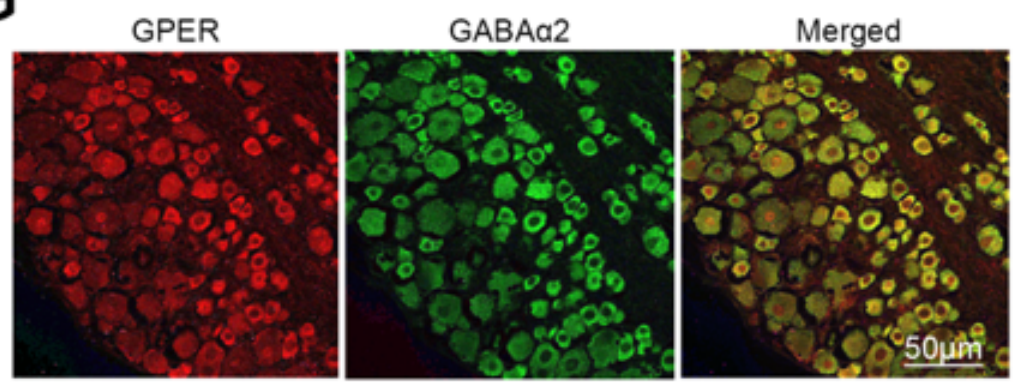

\section{Figure 3}

GPER contributes to spared nerve injury (SNI)-induced pain behaviours in the dorsal root ganglion (DRG), but not the spinal dorsal horn (SDH). (A, B) Intrathecal injection of the $G$ protein-coupled estrogen receptor-1 agonist G1 $(0.2 \mu \mathrm{g} / \mu \mathrm{l}, 0.5 \mu \mathrm{l} /$ hemisphere), the estrogen receptor-a agonist PPT $(0.02 \mathrm{ng} / \mu \mathrm{l}, 0.5$ $\mu \mathrm{l} /$ hemisphere $)$ and the estrogen receptor- $\beta$ agonist DPN $(0.02 \mathrm{ng} / \mu \mathrm{l}, 0.5 \mu \mathrm{l} /$ hemisphere), directly into the subarachnoid space induced mechanical allodynia and cold hyperalgesia. $* \star * \mathrm{P}<0.001$ versus vehicle 
(10\% dimethyl sulfoxide; one-way ANOVA followed by post hoc Dunnett's test); $n=8,8$, and 8 . (C-F) Intraplantar injection of the estrogen receptor- $\beta$ antagonist (MPP; $1 \mathrm{ng} / \mu \mathrm{l}, 1 \mu \mathrm{l}$ ) or the $\mathrm{G}$ protein-coupled estrogen receptor-1 antagonist $(\mathrm{G} 15 ; 1.8 \mu \mathrm{g} / \mu \mathrm{l}, 1 \mu \mathrm{l})$ into the subarachnoid space of SNI rats in day 5 to detect mRNA content of estrogen receptor- $\alpha$, estrogen receptor- $\beta$, and $G$ protein-coupled estrogen receptor and GABAa2 in DRG and SDH (two-way repeated-measures ANOVA); $n=7,7$, and 7. (G) Immunofluorescence co-expression of GPER and GABAa2 in the dorsal root ganglia of rats on day 5 after SNI. Scale bars $=50 \mu \mathrm{m}, \mathrm{n}=6$.
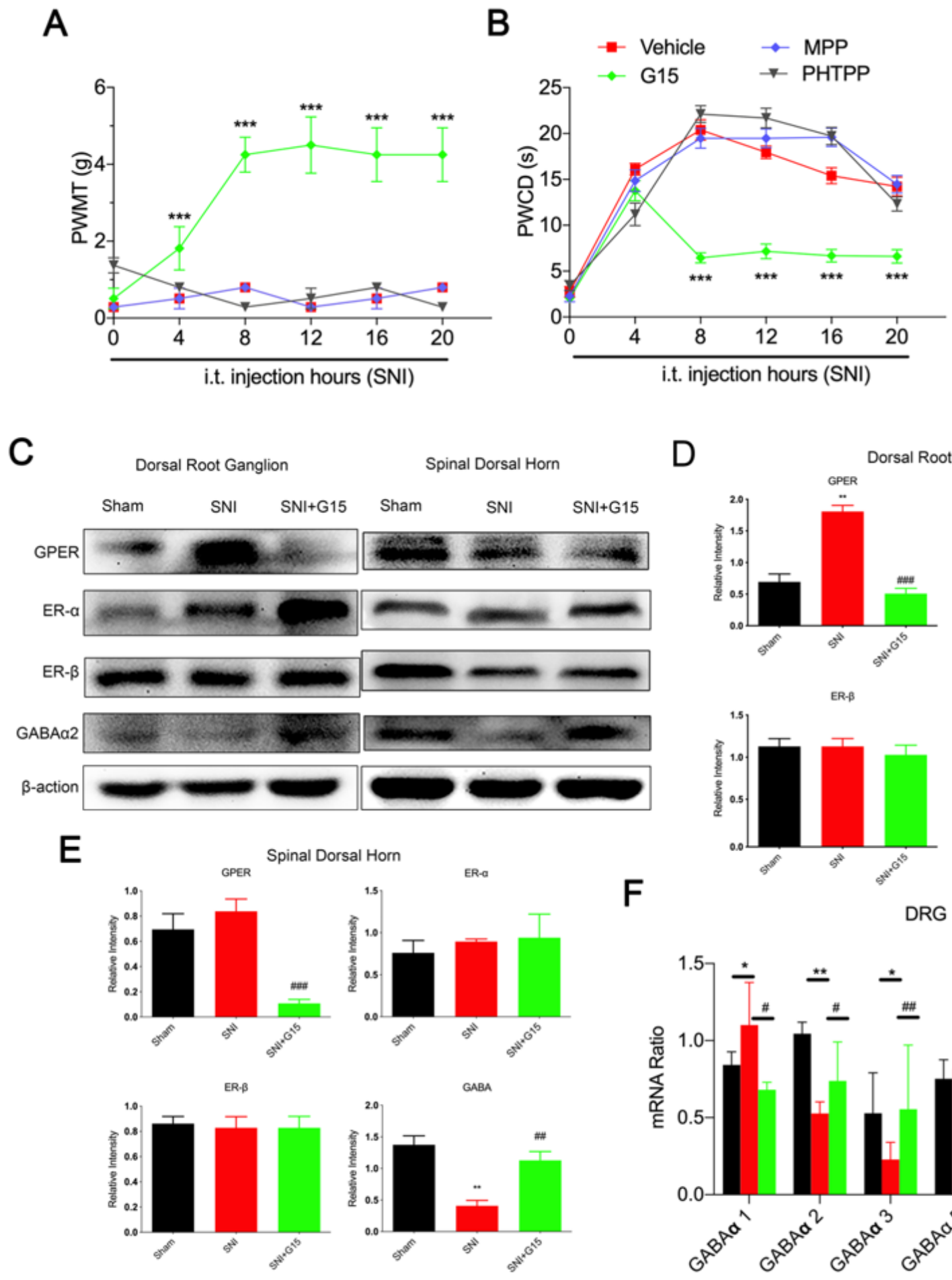

$\mathrm{F}$
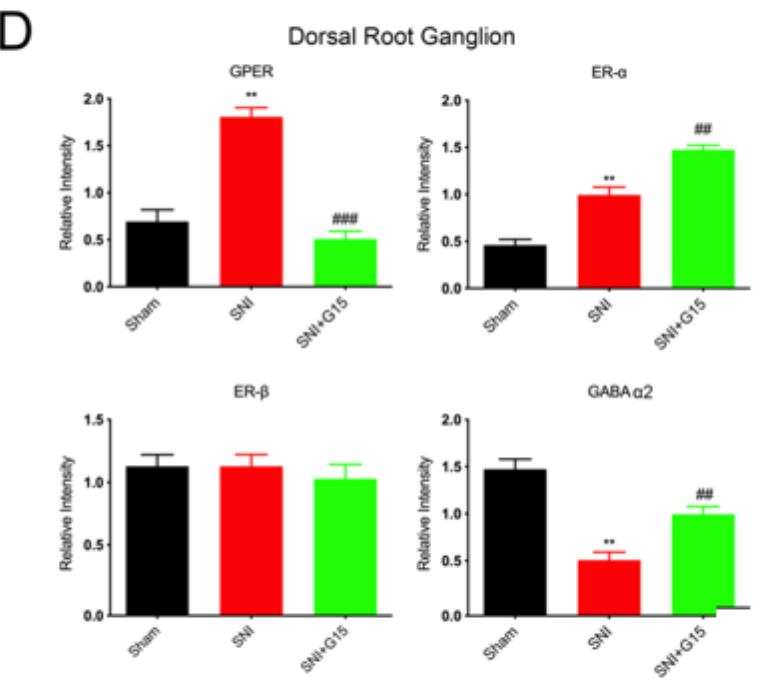

DRG

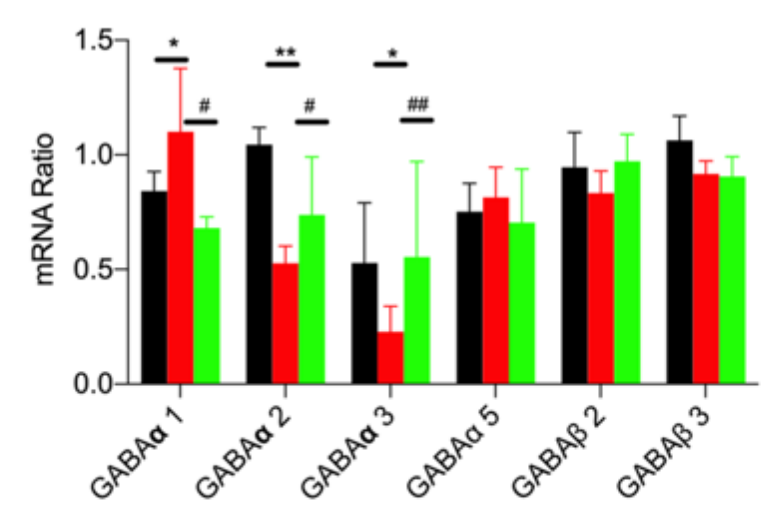




\section{Figure 4}

Inhibiting GPER expression in the dorsal root ganglion (DRG) and spinal cord alleviates spared nerve injury (SNI)-induced pain behaviours in rats and simultaneously restores GABAa2 expression. (A, B) Intrathecal injection of the estrogen receptor- $a$ antagonist (MPP; $1 \mathrm{ng} / \mu \mathrm{l}, 1 \mu \mathrm{l}$ ) or the $\mathrm{G}$ protein-coupled estrogen receptor antagonist (G15; $1.8 \mu \mathrm{g} / \mu \mathrm{l}, 1 \mu \mathrm{l})$ estrogen receptor- $\beta$ antagonist (PHTPP; $1 \mathrm{ng} / \mu \mathrm{l}, 1 \mu \mathrm{l}$ ) into the subarachnoid space of SNI rats in day 5 to detect mechanical allodynia and cold hyperalgesia. ${ }^{*} * \mathrm{P}<0.001$ versus vehicle (10\% dimethyl sulfoxide; one-way ANOVA followed by post hoc Dunnett's test). (C) Western blot images for GPER, ERa, ERß, GABAa2 in the DRG and SDH after Sham, SNI and intrathecal injection of G15 in rats. (D) Both GPER and ERa were increased GABAa2 downregulated in the dorsal root ganglion (DRG) of SNI rats 6 in total. ${ }^{\star \star} \mathrm{P}<0.01$ versus Sham, \#\#P $<0.01$ versus SNI (twotailed Student's t test); $n=3$ and 3. (E) There is no change in each protein in the spinal dorsal horn (SDH) except GABAa2 downregulated. \#\#P $<0.01$ versus SNI (two-tailed Student's t test); $n=3$ and 3. (F) Quantification of the mRNA content of GABA receptor subunits in the DRG defined. Error bars in all panels represent S.E.M. $(n=3$, one-way ANOVA *P $<0.05 ; \star \star P<0.01$ versus Sham; \#\#P $<0.01$ versus $S N I)$. 
A

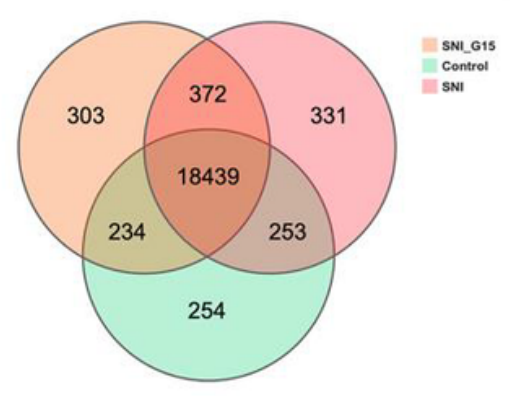

B

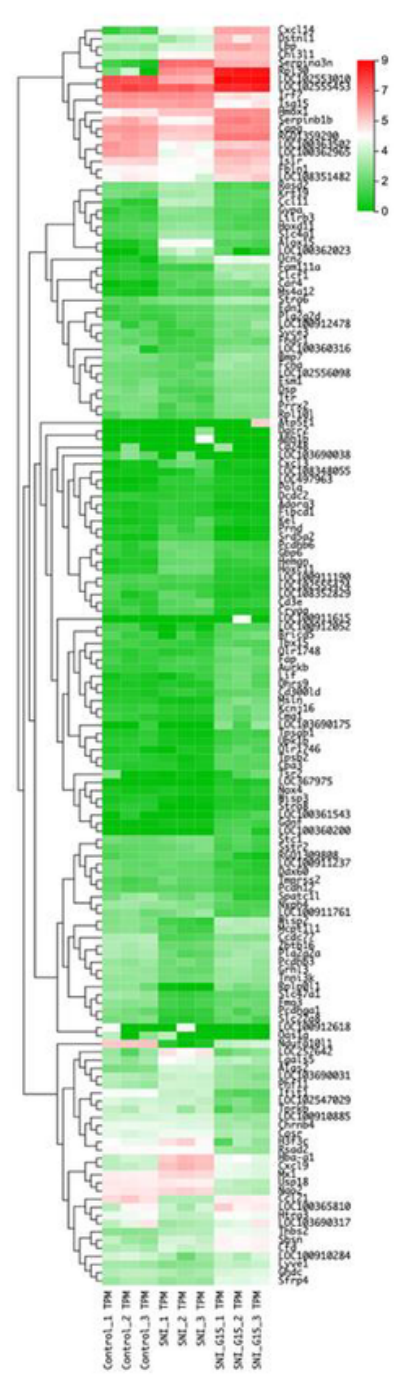

C

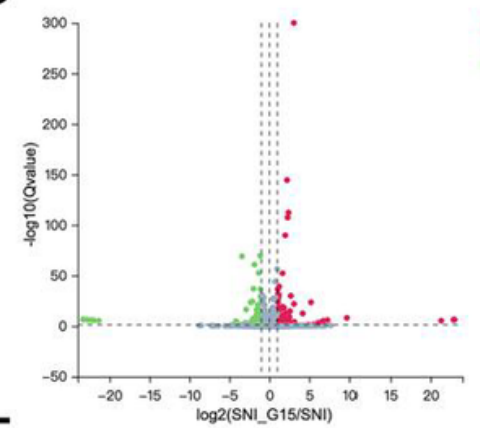

$\mathrm{E}$

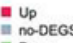

D

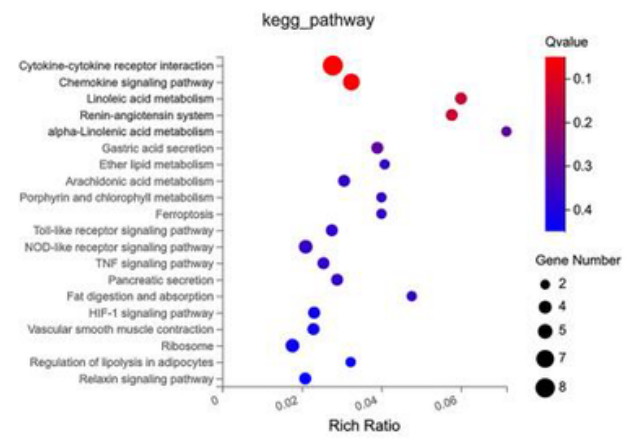

$\mathrm{F}$

up kegg_pathway

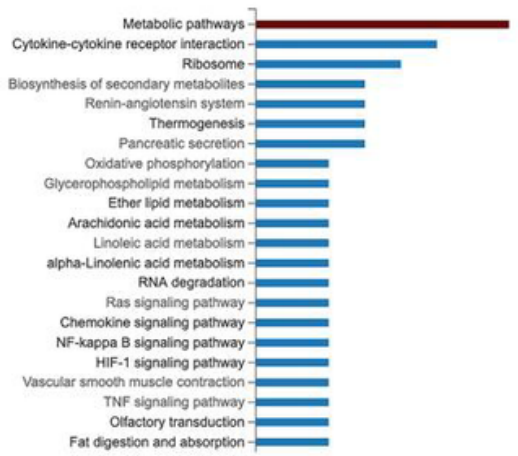

Down kegg_pathway

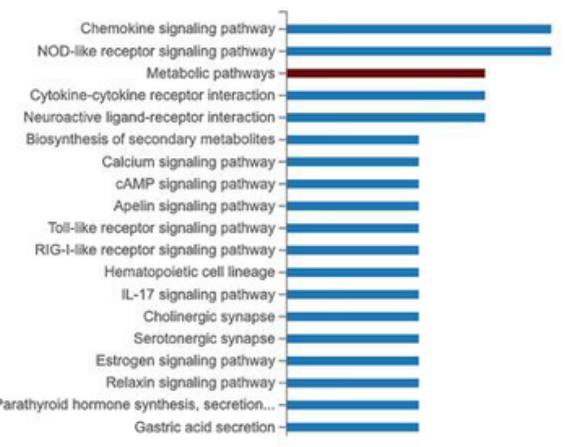

G

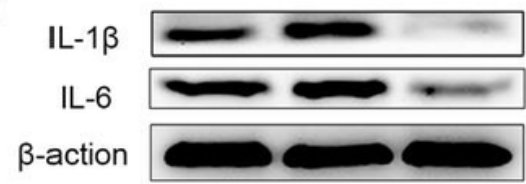

IL-6

IL-1 $\beta$
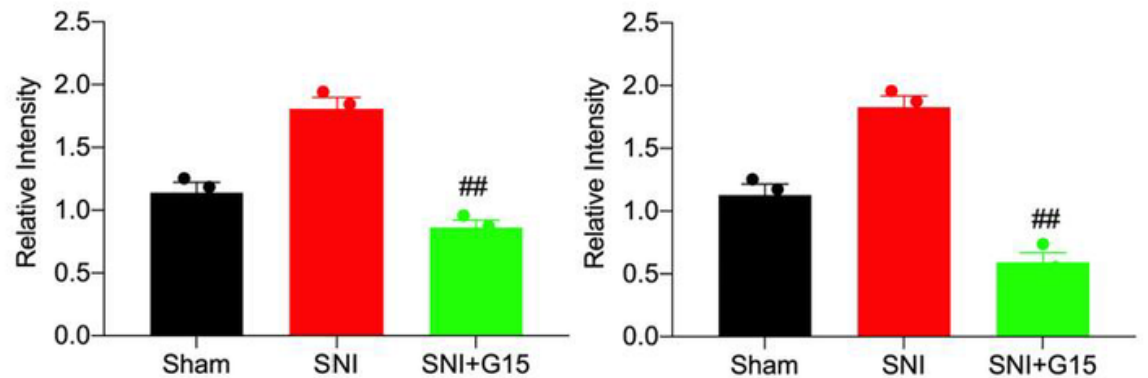

\section{Figure 5}

RNA-Seq identifies mRNA expression changes in the dorsal root ganglion (DRG) of spared nerve injury (SNI) rat models and G15-treated rats, and inflammation occurs synchronously. (A) Venn diagram showing the overlapping of DEGs in DRG from Sham, SNI, SNI+G15 rats. (B) Heat map illustrations of hierarchical clustering analysis of differently expressed mRNA (DEmRNA) in DRG from Sham, SNI, SNI+G15 group rats. (C) Volcano plots of DEmRNAs in DRG of SNI+G15 group rats vs. SNI rats. Red points refer to upregulated DEGs and green points refer to downregulated DEGs, whereas grey spots 
indicate non-DEGs. (D) Bubble plots showing the top 20 significant pathways for upregulated DEmRNAs. And downregulated DEmRNAs. (E, F) KEGG pathway analysis of DEmRNAs. E, The top 22 significant biological processes, molecular functions, and cellular components of upregulated DEmRNAs. F, The top 19 significant biological processes, molecular functions, and cellular components of downregulated DEmRNAs. The dotted line indicated a p value $<0.001$. (G) Protein expression levels of IL-1 $\beta$ and IL- 6 in DRG from Sham, SNI, SNI+G15 rats. $\left(n=3\right.$, one-way ANOVA, ${ }^{\star *} P<0.01$ versus Sham; \#\#P $<0.01$ versus $\mathrm{SNI})$.

A

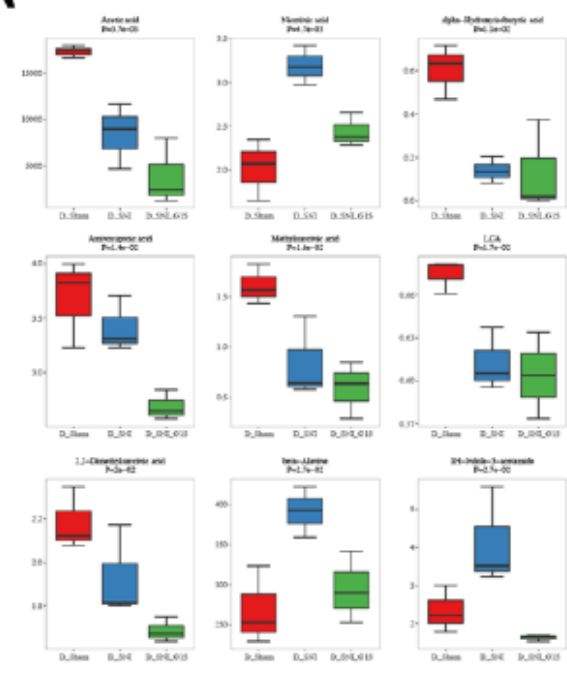

B

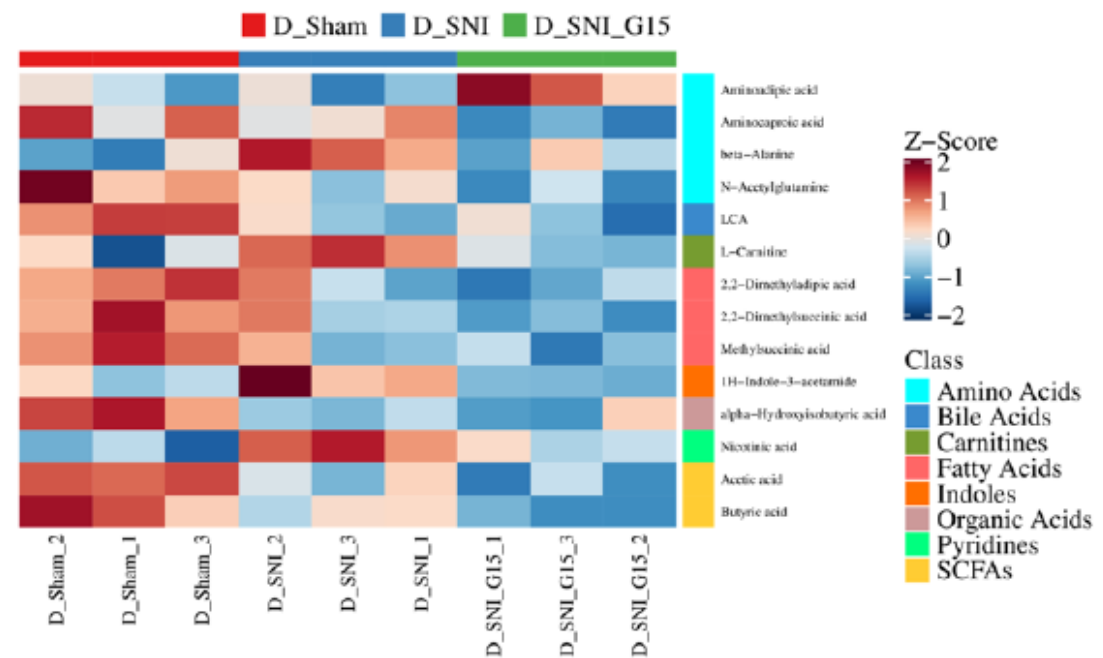

C

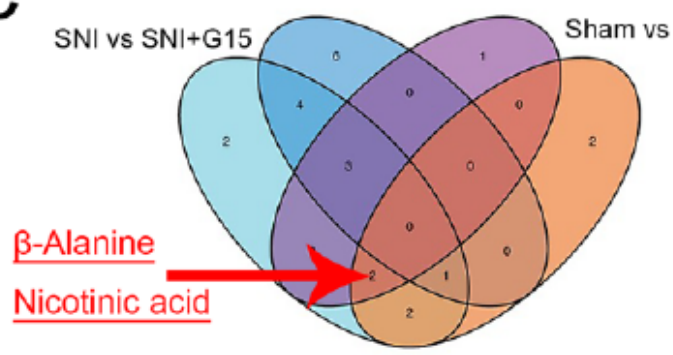

E

Fatty Aei (B) synthesis Pyrimidiecoletubolism

D
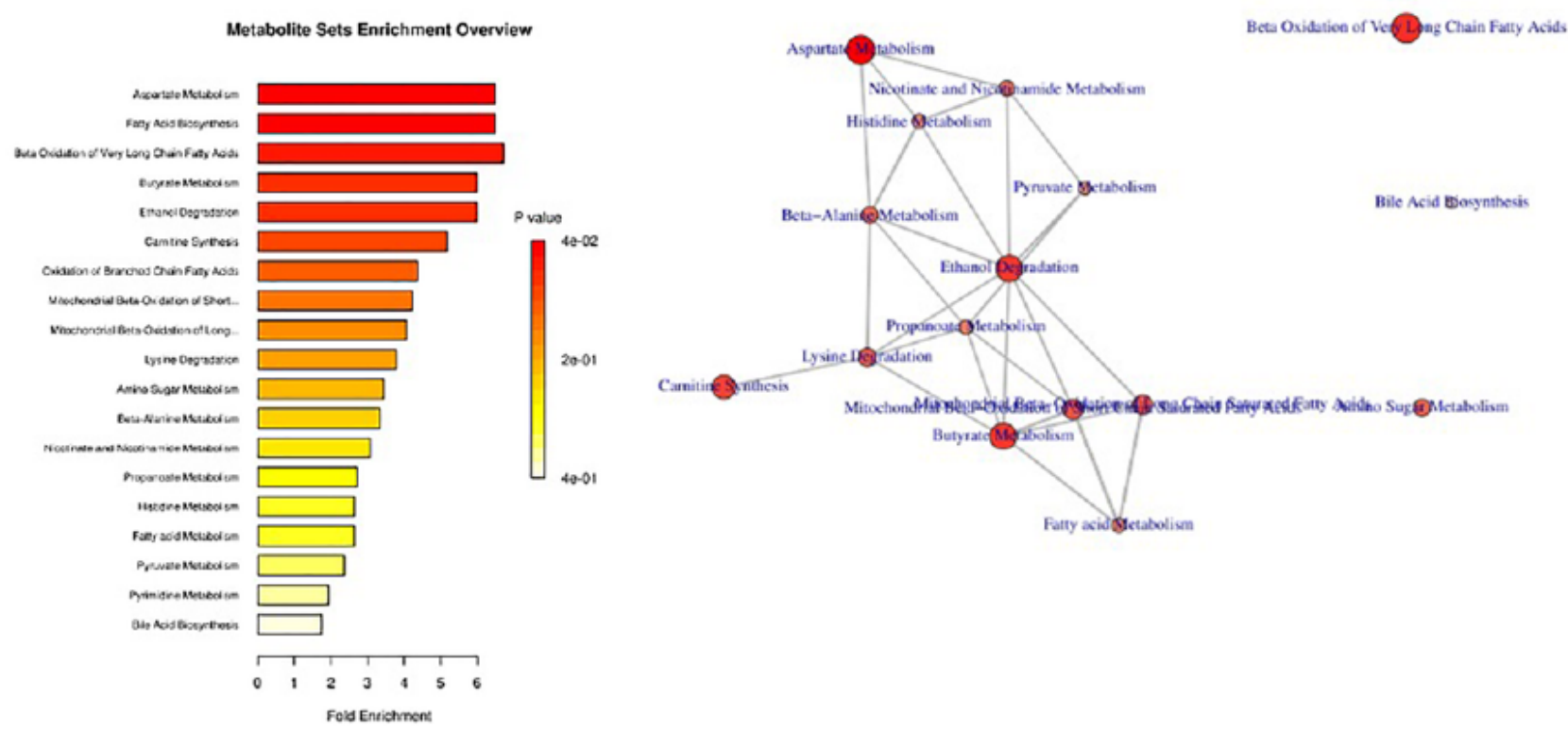


\section{Figure 6}

Metabolomics reveal that $\beta$-alanine metabolism is involved in post-spared nerve injury (SNI) GPER upregulation. (A) Box charts of the relative trends of metabolites involved in the important metabolic pathways that were identified. Data are expressed as the mean \pm SEM. (B) Heatmap of metabolites in DRG of rats in the sham, SNI, andSNI+G15 groups. Red for higher level and blue for lower level. (C) Venn diagram showing the overlapping of metabolites in DRG from Sham, SNI, SNI+G15 rats pairwise comparison. The number represents the number of metabolites that change together in a pairwise comparison. (D) Metabolites sets enrichment overview analysis. (E) PPI network analysis of metabolites involved in GPER response-related pathways in DRG of SNI model rats. metabolites related with pain response-related pathways identified by GO and KEGG analysis were subject to PPI network analysis. Bigger circle reflect more interactions and vice versa. 
A
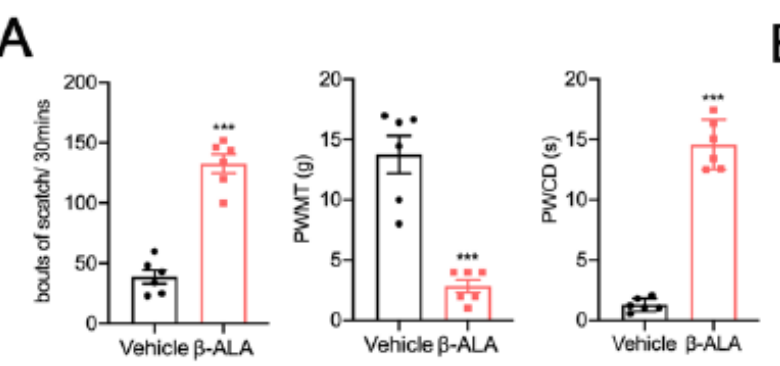

B

C
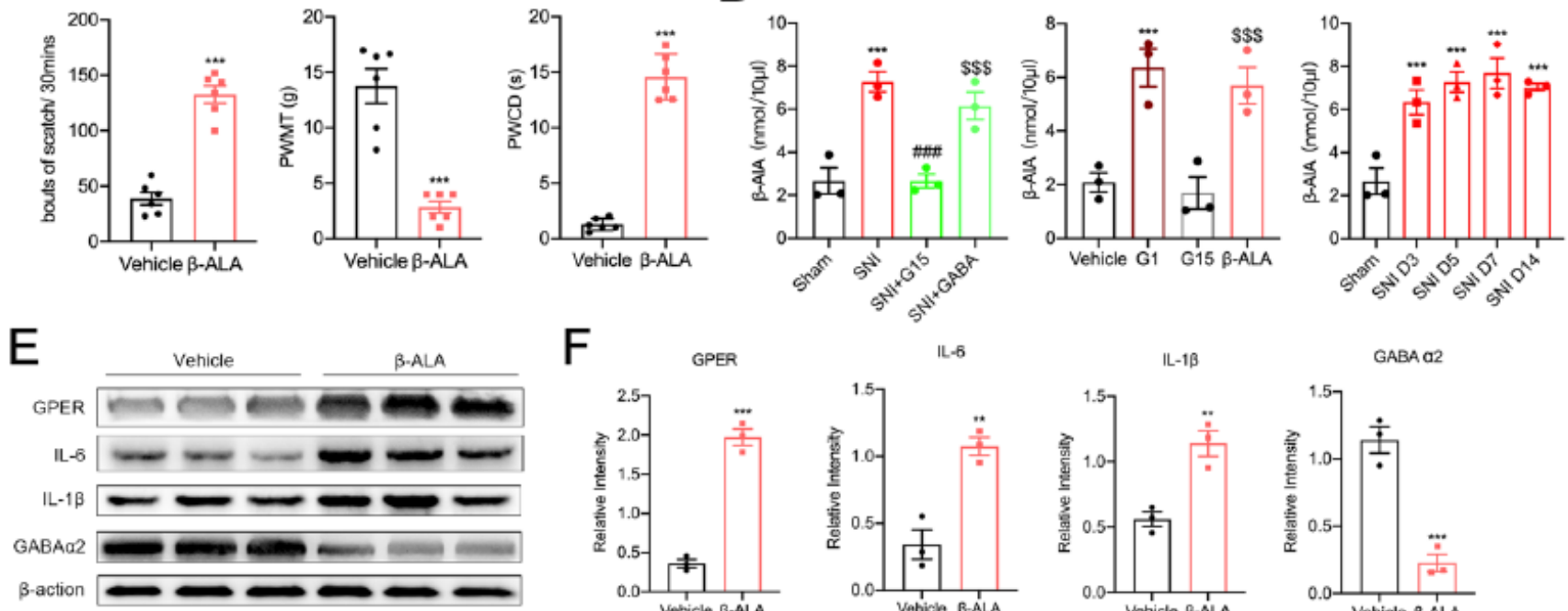

$\mathrm{F}$
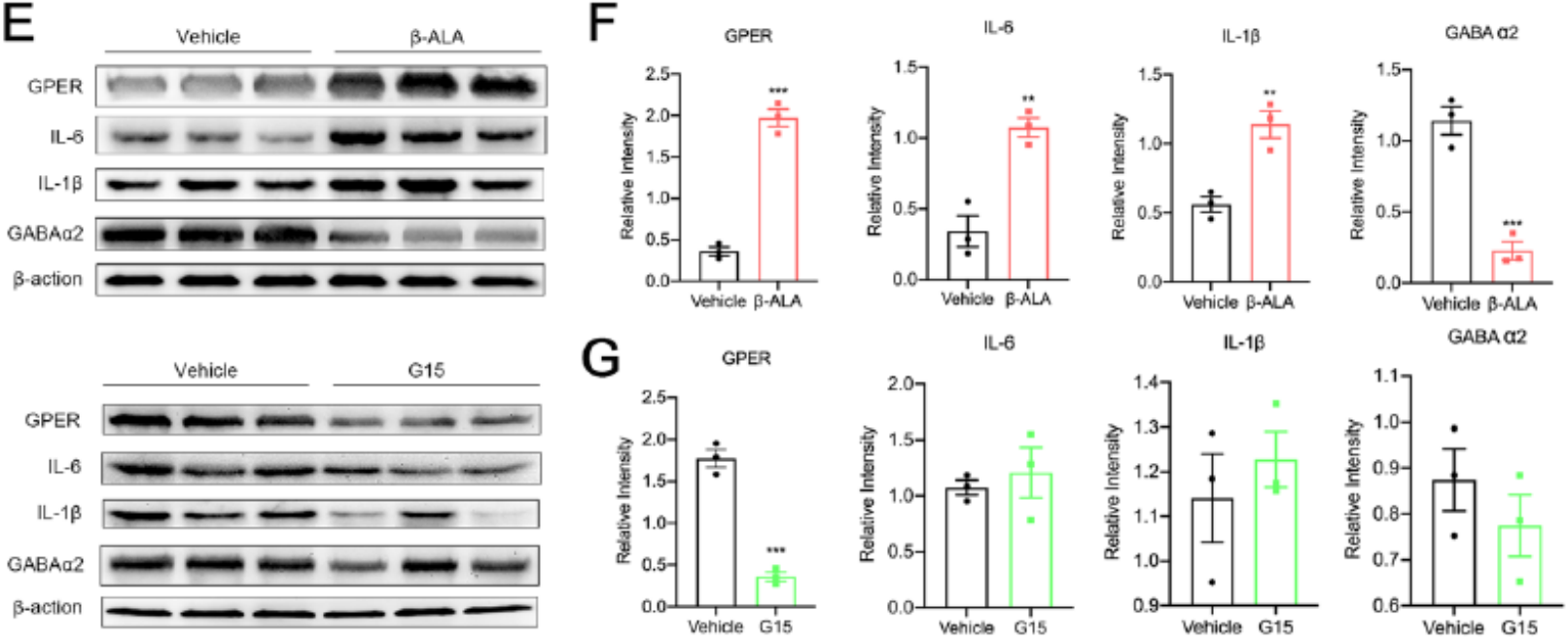

$\mathrm{H}$
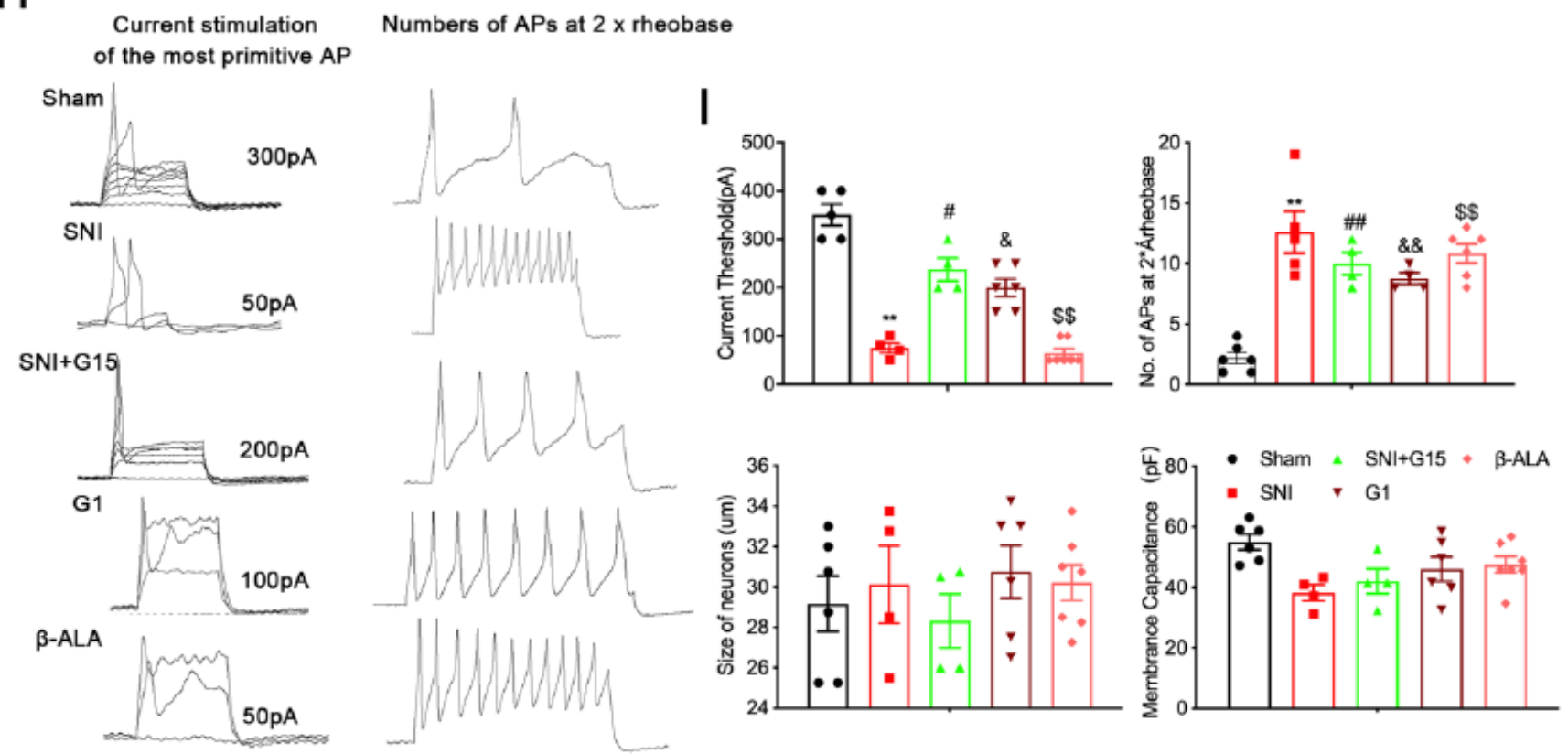

\section{Figure 7}

GPER/ $\beta$-alanine positive feedback interaction in the dorsal root ganglion (DRG) mediating neuronal sensitisation and neuroinflammation responses. (A) Itch allodynia and mechanical allodynia and cold hyperalgesia were developed in intrathecal $\beta$-alanine rat after $24 h(n=8)$. (B) $\beta$-alanine content in DRG of rats in the Sham, SNI, and SNI+G15, SNI+GABA groups. (C) $\beta$-alanine content in DRG of rats in the Vehicle, G1, and G15 groups. (D) $\beta$-alanine content in DRG of rats in the Sham, SNI day 3, 5, 7, 14 groups. 
(two-tailed Student's $t$ test); $n=3$. (E) Western blot images for GPER, ERa, ER $\beta, I L-6, I L-1 \beta, G A B A a 2$ in the DRG after intrathecal injection of $\mathrm{G} 1$ and $\beta$-alanine in rats. (F) Both GPER, IL-6, IL-1 $\beta$ were increased, GABAa2 downregulated in the dorsal root ganglion (DRG) of G1 group rats 6 in total. ${ }^{* * P}<0.01, * \star * P<$ 0.001 versus Vehicle, (two-tailed Student's t test); $n=3$ and 3. (G) Both GPER, IL-6, IL-1 $\beta$, GABAa2 had no significant. $(\mathrm{H})$ Current threshold (rheobase) was determined as the current required for activating the first action potential. On the right, representative traces of action potentials (APs) evoked by current injections into DRG neurons from Sham, SNI, SNI+G15, G1, $\beta$-alanine groups; $n=4-6$ neurons per group; $O n$ the right, twice in the Fig., the number of action potentials produced at the corresponding $2 \times$ rheobase. (I) Statistical analysis revealed the rheobase; Size of neurons; Membrane capacitance; Number of action potentials (APs) at $2 \times$ rheobase in DRG neurons. $n=4-6$ neurons per group, $\star \star P P<0.01$ versus Sham; \#P< 0.05 , \#\#P $<0.01$ versus SNl; \&P $<0.05, \& \& \mathrm{P}<0.01$ versus Sham; $\$ \mathrm{P}<0.01$ versus Sham, by two-tailed unpaired Student's t-test.

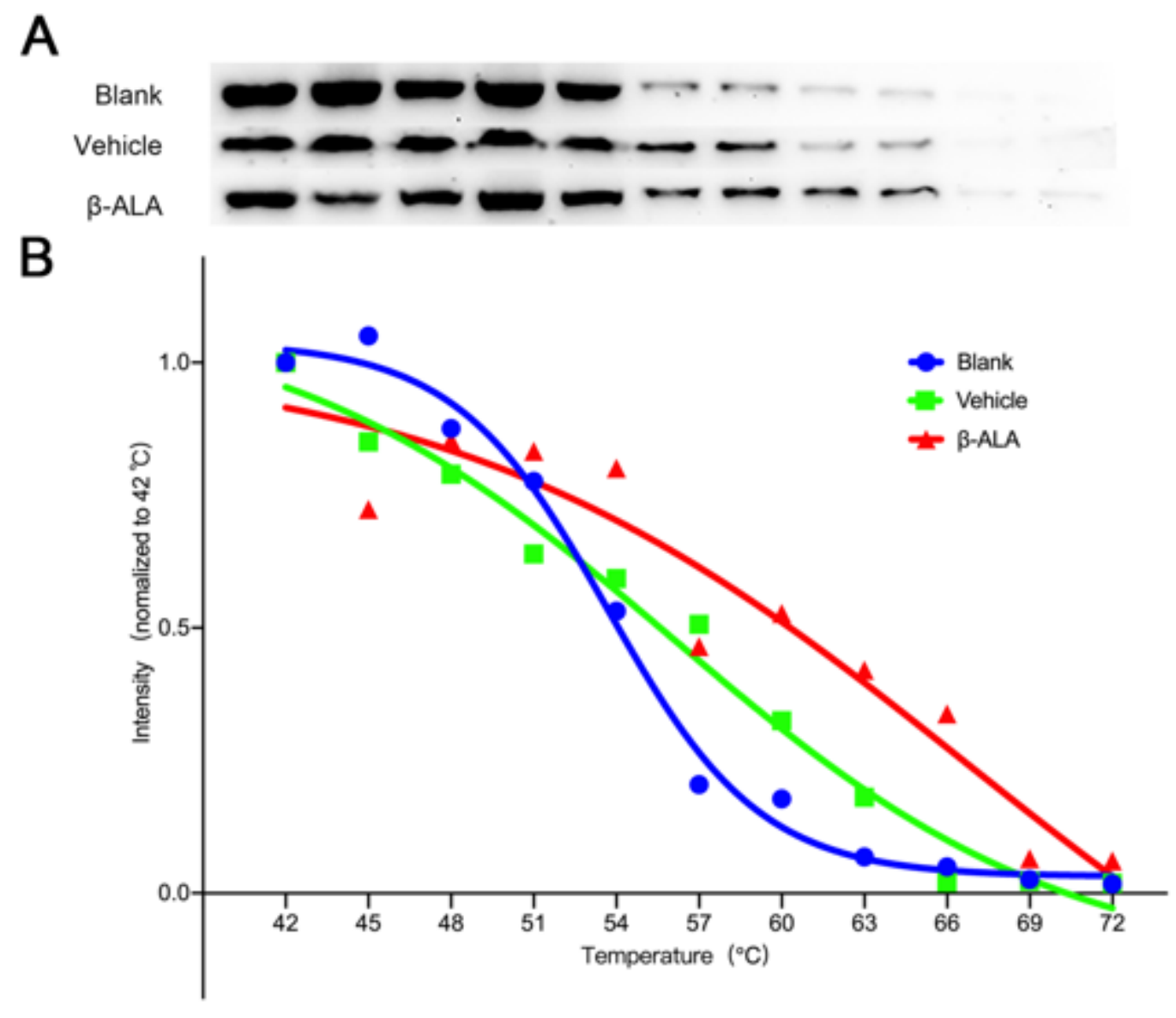

Figure 8

$\beta$-alanine binding to GPER in dorsal root ganglion (DRG) cells in vitro. (A) Representative immunoblotting analysis of the protein lysates of DRG neurons isolated from blank control, Vehicle, $\beta$-Alanine rats DRG tissues and heated at a series of temperatures from 42 to $72^{\circ} \mathrm{C}$ for 3 minutes. (B), Statistical regression (4 parameter logistic regressions, $\mathrm{P}<0.05$ ) of separate immunoblots generating the cellular thermal shift assay curves for theenhanced thermal stability of GPER by $\beta$-Alanine. 


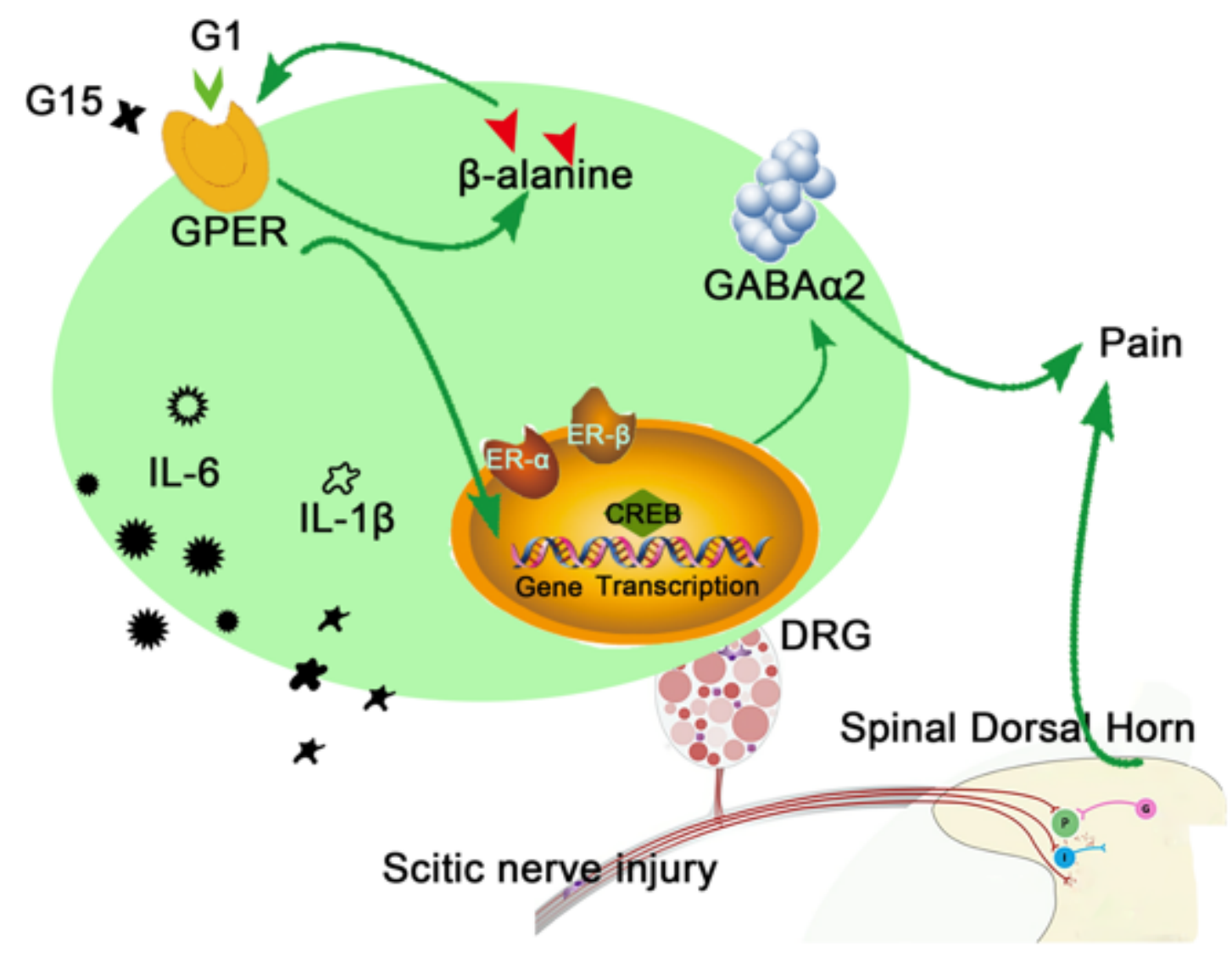

Figure 9

The mechanisms diagram of GPER/ $\beta$-alanine mediating neuronal sensitisation and neuroinflammation in modulating neuropathic pain. Show that upregulating GPER in DRG cells signals to sensory neurons upon peripheral nerve damage, inducing neuronal $\beta$-Alanine increase while positive promote GPER, the combined effect of them further activates IL-1 $\beta$ and IL- 6 , regulates GABAa2 in the DRG. The GPER/ $\beta$ Alanine positive interaction in DRG uncovers enhances pain sensation and promotes chronic pain development. 